\title{
Meteorites and the Chemical Evolution of the Milky Way
}

\author{
Larry R. Nittler \\ Carnegie Institution of Washington \\ Nicolas Dauphas \\ The University of Chicago
}

\begin{abstract}
The theory of galactic chemical evolution (GCE) describes how the chemical and isotopic composition of galaxies changes with time as succeeding generations of stars live out their lives and enrich the interstellar medium with the products of nucleosynthesis. We review the basic astronomical observations that bear on GCE and the basic concepts of GCE theory. In addition to providing a standard set of abundances with which to compare GCE predictions, meteorites also provide information about how the galaxy has evolved through the study of preserved presolar grains and radioactive isotopes.
\end{abstract}

\section{INTRODUCTION}

The solar system is situated in the disk of the Milky Way galaxy, some 8.5 kiloparsecs from the galactic center. It formed 4.567 G.y. ago (Amelin et al., 2002) and its composition represents a snapshot of the composition of the Milky Way in the solar neighborhood at that time. The bulk composition of the solar system has often been referred to as the "cosmic composition" (Anders and Grevesse, 1989), with the underlying assumption that it represents the average composition of the galaxy. However, stellar nucleosynthesis is a phenomenon that is discrete in both time and space. The concept of cosmic composition therefore breaks down when the chemical and isotopic composition of the galaxy is examined at a fine scale and astronomy abounds with examples of objects and environments with nonsolar compositions. Galactic chemical evolution (GCE) is the name given to the theory of how the chemical composition of a galaxy varies with time and space as succeeding generations of stars live out their lives and enrich the interstellar medium (ISM) with the products of nucleosynthesis. Note that the word "chemical" is somewhat misleading in this context, since GCE refers only to the abundances of nuclei in the galaxy, not to the chemical state in which they might appear.

In this chapter, we discuss some of the ways in which meteorites can help unravel the presolar nucleosynthetic history of the Milky Way. A key constraint for models of GCE has long been the solar isotopic and elemental abundance pattern, largely determined by measurements of CI chondrites. However, meteorites also preserve a record of GCE in the form of preserved presolar dust grains and extinct radioactivities. Here, we will review the basic concepts of GCE theory and astronomical constraints before considering the role of GCE in determining the isotopic compositions of presolar grains and the abundances of radioactivities in the early solar system. Although the subject of GCE has a rich history, we concentrate on recent developments of the field. The reader is referred to the excellent books by Pagel (1997) and Matteucci (2003) for comprehensive reviews of the rich astronomical literature on the topic.

\section{BASIC CONCEPTS AND ASTRONOMICAL CONSTRAINTS}

Excluding dark matter, the Milky Way consists of several components, including a thin disk (to which the solar system belongs), a thick disk, a bright inner bulge, and a large spherical diffuse stellar halo. Just how these components formed is not known, but there are several competing models (Matteucci, 2003). Most likely, both primordial collapse of a protogalactic gas cloud and subsequent accretion and merger of smaller systems have played a role (Gibson et al., 2003). However, many aspects of GCE modeling do not depend strongly on the overall model of galactic formation. Because the solar system belongs to the thin disk, we will primarily concentrate on observations and GCE models of this component.

A crucial quantity involved in any discussion of GCE is metallicity, which is the abundance of elements heavier than $\mathrm{He}$ ("metals" for astronomers). The letter $\mathrm{Z}$ is usually used to indicate the total metallicity. However, the normalized Fe abundance $\left([\mathrm{Fe} / \mathrm{H}]=\log (\mathrm{Fe} / \mathrm{H})-\log (\mathrm{Fe} / \mathrm{H})_{\odot}\right)$ is often used as a proxy for total metallicity, as Fe is relatively easy to measure in a large number of astronomical environments. The metallicity of the Sun $\left(\mathrm{Z}_{\odot}\right)$ has long been thought to be $\sim 2 \%$, but a recent downward revision in the solar $\mathrm{O}$ abundance by Allende Prieto et al. (2001) now indicates that it is closer to $1.4 \%$.

Descriptions and models of GCE require a number of key ingredients:

1. Boundary conditions: The initial composition (often taken to be that generated by the Big Bang) and whether the system is closed or open must be defined. 
2. Stellar yields: The abundances of isotopes produced by nucleosynthesis in stars of various types are required. These are determined by stellar evolutionary calculations coupled to nuclear reaction networks (e.g., Meyer and Zinner, 2006). In general, the nucleosynthesis abundance patterns ejected by stars depend critically on the stellar mass and metallicity. Moreover, there are still large uncertainties in the predicted yields due to uncertainties in the stellar evolution physics and nuclear reaction cross sections. A key concept related to stellar yields is the definition of primary and secondary species. A primary specie is one that can be synthesized in a zero-metallicity star, consisting initially of pure $\mathrm{H}$ and $\mathrm{He}$. Examples of primary species are ${ }^{16} \mathrm{O}$ and ${ }^{12} \mathrm{C}$, both made by stellar He burning. In contrast, nucleosynthesis of a secondary specie requires some preexisting metals to be present in the star. Some secondary species include the heavy isotopes of $\mathrm{O},{ }^{14} \mathrm{~N}$, and the $s$-process elements (Meyer and Zinner, 2006).

3 . The star formation rate (SFR) is usually parameterized in GCE models. A very common parameterization is to assume that the SFR is proportional to the disk gas surface density $\left(\sigma_{\mathrm{g}}\right)$ to some power: $\Psi \propto \sigma^{\mathrm{n}}$, where $\mathrm{n}=1-2$ (Schmidt, 1959). However, there are many examples of more complicated expressions for the SFR (e.g., Dopita and Ryder, 1994; Wyse and Silk, 1989).

4. The initial mass function $[\phi(\mathrm{m})]$ describes the number distribution of stars that form in a given mass interval at a given time. It is usually parameterized as a single- or multicomponent power law; for example, the common Salpeter (1955) IMF is: $\phi(M) \propto M^{-2.35}$. Other parameterizations have different power-law indices for different mass ranges (Scalo, 1986). In most models it is assumed that the IMF is constant in space and time; this is consistent with observational evidence (Kroupa, 2002).

5. Except for the simplest models (see next section), infall of gas from the halo onto the disk and outflows from galactic winds are often included in GCE models. The infall rate is usually assumed to vary with galactocentric radius and decrease with time. There is evidence for at least two independent episodes of infall leading to the formation of the halo and the thin disk respectively (Chiappini et al., 1997).

Galactic chemical evolution models are constrained by a number of disparate observational data. These include the present-day values of the star formation and supernova rates, the present-day values of the surface mass density of stars and gas (Fig. 1), stellar mass function, and gas infall rate, as well as chemical abundances measured in a wide variety of stars and interstellar gas. The abundance data can be divided into a number of constraints:

1. Solar abundances: The composition of the Sun (e.g., Anders and Grevesse, 1989), determined both by spectroscopy of the Sun and analysis of CI chondrites, represents a sample of the ISM 4.6 G.y. ago and GCE models of the Milky Way disk should reproduce it (Timmes et al., 1995). For many elements and isotopes, the solar system abundances are the only extant data with which to compare GCE models.

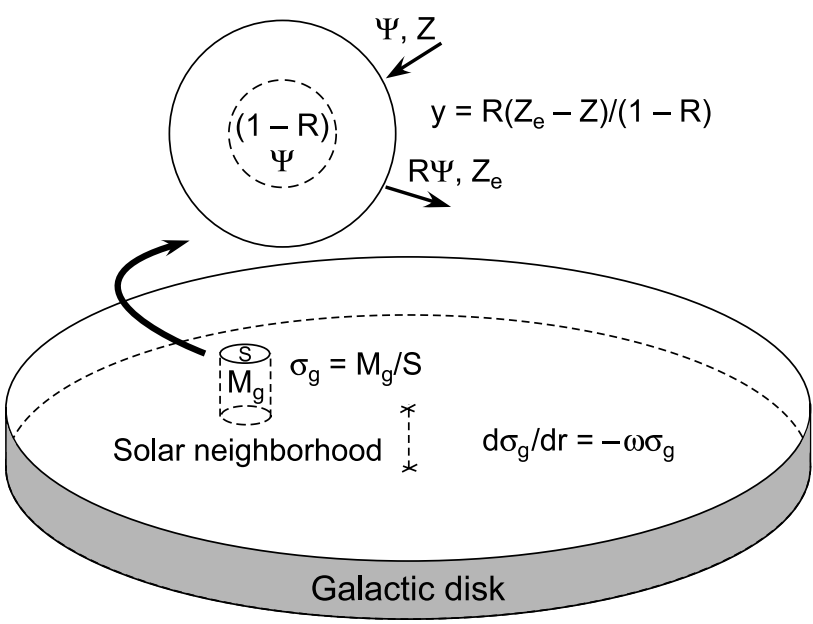

Fig. 1. Closed-box linear model with instantaneous recycling approximation. The gas surface density is the mass of gas contained in a cylinder divided by the area of the top surface of this cylinder. The rate of star formation is assumed to scale linearly with the gas density (linear model) The galactic disk is assumed to have been isolated for most of its history (closed-box model).

2. Age-metallicity relationship (AMR): The measured metallicity of stars decreases on average with stellar age (Twarog, 1980; Edvardsson et al., 1993), as would be expected from the basic idea of GCE. However, there is a large observed scatter in metallicity, greater than a factor of 2 , for stars of a given age in the solar neighborhood (Edvardsson et al., 1993; Rocha-Pinto et al., 2000). This scatter is still not well-explained and makes the AMR a rather weak constraint for GCE models.

3. Abundance ratio evolution: Because different elements are formed by different nucleosynthetic processes, they can evolve at different rates. Thus, studies of element abundance ratios as a function of metallicity can provide important information about GCE (McWilliam, 1997). For example, low-metallicity stars have higher-than-solar ratios of so-called $\alpha$ elements (e.g., ${ }^{16} \mathrm{O},{ }^{24} \mathrm{Mg}$ ) to $\mathrm{Fe}$, but these ratios decrease to solar as solar $[\mathrm{Fe} / \mathrm{H}]$ is reached. This reflects the fact that the $\alpha$ elements are made primarily in Type II supernovae, which evolve rapidly $\left(\leqslant 10^{7} \mathrm{yr}\right)$, whereas a major fraction of $\mathrm{Fe}$ in the galaxy is made by Type Ia supernovae, which evolve on much longer timescales $\left(\sim 10^{9} \mathrm{yr}\right)$. Thus, high $\alpha / \mathrm{Fe}$ ratios at low metallicities indicate that Type Ia supernovae had not yet had time to evolve and enrich the ISM with their ejecta. The exact shape of abundance trends are determined by the relative fractions and timescales of Type Ia and II supernovae as well as other details of GCE.

4. G-dwarf metallicity distribution: $\mathrm{G}$ dwarfs are lowmass stars $\left(\sim 0.9-1.1 \mathrm{M}_{\odot}\right)$ that have lifetimes greater than or equal to the age of the disk. These stars are not active sites of nucleosynthesis and the compositions of their envelopes reflect the compositions of the interstellar gas from which they formed. Thus, their metallicity distribution (Fig. 2) represents a history of the star formation rate since the Milky 
Way formed (van den Bergh, 1962; Schmidt, 1963; Jorgensen, 2000). As discussed below, the simplest closed-box GCE models overpredict the number of low-metallicity $\mathrm{G}$ dwarfs. G dwarfs are actually massive enough that some of them have begun to evolve away from the main sequence, which requires that a correction be applied to the metallicity distribution. Note, however, that stars with lower masses, such as K or M dwarfs, show the same discrepancy between the observed and the predicted abundance of metal deficient stars (Kotoneva et al., 2002). Various approaches have been adopted for solving this problem, including preenrichment of the gas, varying initial mass function, or gas infall. Among these, infall of low-metallicity gas on the galactic plane is the most likely culprit. At present, so-called high-velocity clouds are seen falling on the galactic disk. Some of these clouds have the required low metallicity (down to $\sim 0.1 \mathrm{Z}_{\odot}$ ) to solve the G-dwarf problem (Wakker et al., 1999).

5. Abundance gradients: Observations of abundances in molecular clouds, stars, and planetary nebulae at a range of distances from the galactic center indicate the presence of metallicity gradients, where the inner galaxy is more metalrich than the outer galaxy (Matteucci, 2003). This result indicates that the Milky Way disk formed in an inside-out fashion, with the inner disk forming on a shorter timescale than the outer disk. The precise values for metallicity gradients expected for the disk depend strongly on the balance between the radial dependences of the SFR and the infall rate.

\section{GALACTIC CHEMICAL EVOLUTION MODELS}

With the ingredients and observational constraints described in the previous section, models of GCE can be con-

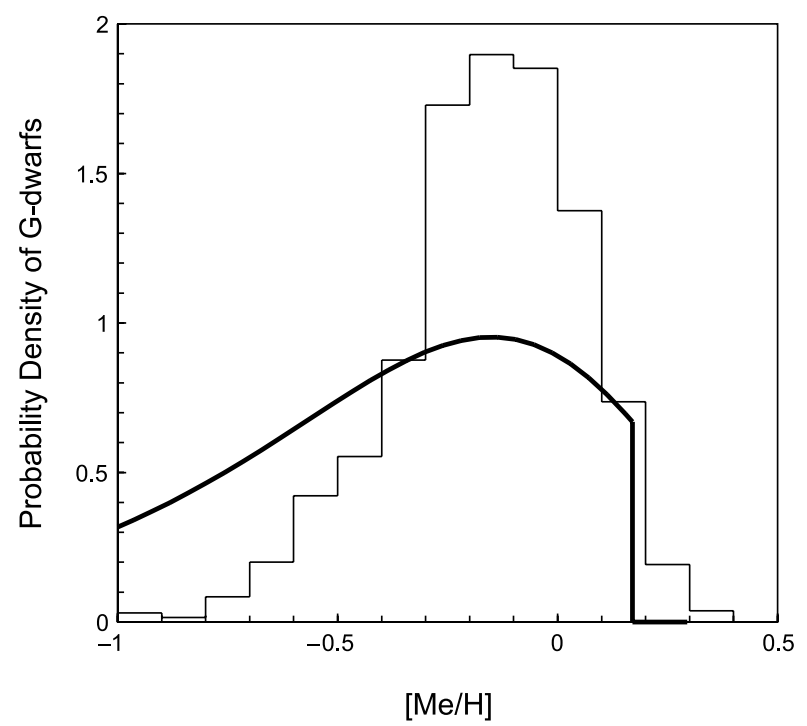

Fig. 2. G-dwarf metallicity distribution in the solar neighborhood. Metallicity is expressed as $[\mathrm{Me} / \mathrm{H}]=\log (\mathrm{Me} / \mathrm{H})-\log (\mathrm{Me} / \mathrm{H})_{\odot}$, where "Me" is a measurable element (usually $\mathrm{O}$ or Fe) Histogram: observations (Nordström et al., 2004). Solid line: closed-box model (Pagel, 1997). structed. We first consider homogeneous models. These (semi-)analytic models make simplifying assumptions that allow the calculation of the mean properties and elemental abundance evolutionary trends of galactic systems. Most homogeneous GCE models of the galactic disk assume cylindrical symmetry and neglect both the finite thickness of the disk and any possible radial flows of matter. Thus, the only relevant spatial variable is the galactocentric radius and what is calculated is the mean abundance evolution within annuli about the galactic center.

We first consider an oversimplified, but illustrative, case: the so-called "simple" model (e.g., Schmidt, 1963; Pagel, 1997; Matteucci, 2003) (Fig. 1). Let us denote $Z_{\mathrm{i}}$ as the mass fraction of nuclide $i$ in the ISM. The galaxy formed from gas of low metallicity $\left(Z_{i 0}=0\right)$. No mass loss or gain occurs during the galactic history (closed-box model). If $E(t)$ is the rate of gas ejection [per unit surface area of the disk (Fig. 1)] from late-evolving stars and $\Psi(t)$ is the rate of gas accretion on nascent stars, then the rate of change of the gas surface density can be written as

$$
\mathrm{d} \sigma_{\mathrm{g}} / \mathrm{dt}=\mathrm{E}-\Psi
$$

The rate of change of the abundance of any nuclide $i$ is

$$
\mathrm{d}\left(\mathrm{Z}_{\mathrm{i}} \sigma_{\mathrm{g}}\right) / \mathrm{dt}=\mathrm{Z}_{\mathrm{i}, \mathrm{e}} \mathrm{E}-\mathrm{Z}_{\mathrm{i}} \Psi-\mathrm{Z}_{\mathrm{i}} \sigma_{\mathrm{g}} / \tau
$$

where $Z_{i, e}$ is the mass fraction of $i$ in the ejecta of all stars and $\tau$ is the mean-life (if the nuclide is radioactive). There is no delay between accretion of gas in stars and return of the nucleosynthetically enriched gas to the ISM (instantaneous recycling approximation). This assumption allows us to write $\mathrm{E}(\mathrm{t})=\mathrm{R} \Psi(\mathrm{t})$, where $\mathrm{R}$ is the so-called "return fraction," the rate at which mass is returned to the ISM. That is, as soon as a generation of stars is formed, a fraction $\mathrm{R}$ instantaneously comes back out, and a fraction $(1-R)$ remains locked up in stars (white dwarfs, etc.), which do not return mass to the ISM.

After some rearrangement and combining equations (1) and (2), it is straightforward to show that

$$
\mathrm{d} \mathrm{Z}_{\mathrm{i}} / \mathrm{dt}=\mathrm{R} \Psi\left(\mathrm{Z}_{\mathrm{i}, \mathrm{e}}-\mathrm{Z}_{\mathrm{i}}\right) / \sigma_{\mathrm{g}}-\mathrm{Z}_{\mathrm{i}} / \tau
$$

Defining the yield y as the quantity of newly synthesized matter per unit mass of stellar remnants

$$
\mathrm{y}=\mathrm{R}\left(\mathrm{Z}_{\mathrm{i}, \mathrm{e}}-\mathrm{Z}_{\mathrm{i}}\right) /(1-\mathrm{R})
$$

and assuming a metallicity-independent yield $\left(\mathrm{Z}_{\mathrm{i}, \mathrm{e}}-\mathrm{Z}_{\mathrm{i}}\right.$ is constant) and a linear star formation rate

$$
\Psi=\omega \sigma_{\mathrm{g}} /(1-\mathrm{R})
$$

it follows that

$$
\mathrm{dZ}_{\mathrm{i}} / \mathrm{dt}=\mathrm{y} \omega-\mathrm{Z}_{\mathrm{i}} / \tau
$$


This equation can be integrated by the method of varying constant

$$
\mathrm{Z}_{\mathrm{i}}=\mathrm{y} \omega \tau\left(1-\mathrm{e}^{-\mathrm{t} / \tau}\right)
$$

In the limit of a stable nuclide ( $\tau \rightarrow \infty$ ), the previous equation assumes the form

$$
\mathrm{Z}_{\mathrm{i}}(\tau \rightarrow \infty)=\mathrm{y} \omega \mathrm{t}
$$

An immediately obvious result is that the simple model predicts that the abundance of a stable primary isotope (whose yield y is independent of time and metallicity) increases linearly with time. Moreover, and of great importance to the interpretation of presolar grain data (section 6), the simple model also predicts that the ratio of a secondary isotope to a primary one increases linearly with total metallicity (Pagel, 1997). Note that a great deal of physics is hidden by the formulation above. For example, actual calculation of y requires integrating nucleosynthetic yields of stars of different mass over the initial mass function. We will revisit the linear closed-box model and its predictions for radioactive nuclei in section 4 .

The main virtue of this model is its simplicity. However, the simple model fails to explain the G-dwarf metallicity distribution (Fig. 2) in that it predicts far more metal deficient stars than what is observed. This is known as the Gdwarf problem. As discussed in the previous section, it now appears most likely that the discrepancy is due to the closedbox assumption; gas flows, especially infall of low-metallicity gas on the disk, must be included in GCE models. Beyond the G-dwarf problem, the simple model also breaks down when trying to describe the evolution of elements produced by long-lived stars, for which the instantaneous recycling approximation is not valid. Moreover, the observed age-metallicity relationship for stars in the solar neighborhood is not well described by equation (8). Thus, more realistic models of GCE are needed. Including infall in an analytic GCE model requires parameterization of the infall rate (e.g., Larson, 1974; Lynden-Bell, 1975). Clayton (1985) provided a very flexible family of GCE models, which he called the "standard model." These models are exactly soluble and allow great freedom in parameterizing both the star formation rate and the infall rate. Clayton's standard model is very useful for understanding the physical behavior of galactic gas without resorting to numerical calculations. Pagel (1989) modified Clayton's model to include a fixed time delay for elements produced by long-lived stars (e.g., Fe, s-process elements).

Although sophisticated analytical GCE formalisms, like those of Clayton (1985) and Pagel (1989), are very useful for providing gross physical understanding of GCE, more realistic models require complete relaxation of the instantaneous recycling approximation and closed-box assumptions. Such models do not usually have analytical solutions and require numerical calculation. Typical formalisms (e.g., Matteucci and Greggio, 1986; Timmes et al., 1995) require solving coupled integro-differential equations with separate terms describing ISM enrichment by stellar ejecta, star formation, infall, outflow and radioactive decay, respectively

$$
\begin{array}{r}
\frac{d}{d t} \sigma_{i}(r, t)=\int_{M_{L}}^{M_{U}} \psi\left(r, t-\tau_{m}\right) X_{m i}\left(t-\tau_{m}\right) \phi(m) d m \\
-Z_{i}(r, t) \psi(r, t) \\
+\frac{d}{d t} \sigma_{i}(r, t)_{\text {infall }}-\frac{d}{d t} \sigma_{i}(r, t)_{\text {outflow }} \\
-\sigma_{i}(r, t) / \tau_{i}
\end{array}
$$

where $\sigma_{i}(r, t)$ is the surface mass density of gas in the form of isotope $i$ at galactocentric radius $r$ and time $t ; M_{L}$ and $\mathrm{M}_{\mathrm{U}}$ are the lower and upper mass limits, respectively, of stars that enrich the ISM at a given time; $\psi$ is the star formation rate; $X_{m i}$ is the mass fraction of $i$ ejected by a star of mass $\mathrm{m} ; \phi$ is the initial mass function; $\tau_{\mathrm{m}}$ is the lifetime of a star of mass $m ; Z_{i}(t)$ is the mass fraction of $i$ in the ISM at time $t$; and $\tau_{\mathrm{i}}$ is the mean lifetime for isotope $\mathrm{i}$. In fact, the first term is often divided into separate integrals for the ejecta from single stars and binary stars, since a fraction of the latter will result in supernovae of Type Ia and/or novae (Matteucci and Greggio, 1986; Roman and Matteucci, 2003).

A large number of numerical homogeneous GCE calculations including infall and neglecting the instantaneous recycling approximation have been reported in recent years. Some general conclusions can be drawn from many of these models: (1) The solar abundances of most isotopes up to $\mathrm{Zn}$ can be reproduced to within a factor of 2 (Timmes et al., 1995); in most cases discrepancies are due to uncertainties in the nucleosynthetic processes and yields responsible for the specific isotope. (2) The G-dwarf metallicity distribution can be quite well approximated if the local disk formed by infall of extragalactic gas over a period of several gigayears. (3) The observed abundance ratio trends (e.g., $\mathrm{O} / \mathrm{Fe}$ vs. $\mathrm{Fe} / \mathrm{H})$ are well explained by the time delay between supernovae of Type Ia and Type II. (4) To explain abundance gradients, the disk must have formed inside-out with a strong dependence of the star formation rate on galactocentric radius. Despite the success of modern GCE models in reproducing a large number of observational constraints, it should be remembered that there are still many crucial uncertainties. Of particular concern are remaining uncertainties in the nucleosynthetic yields of many isotopes, especially those of the Fe-peak elements, as well as the precise form and possible spatial or temporal variability of the initial mass function.

The homogeneous models described above explain well many of the average properties of the galaxy, for example, the average element/Fe ratios measured in stars of a given metallicity. However, because of the discrete and stochastic nature of star formation and evolution, local chemical heterogeneities about mean trends are to be expected. Observationally, the scatter in elemental abundances in stars increases with decreasing metallicity. This is especially true for the low-metallicity stars of the galactic halo. Although 
there is a very large scatter in metallicity for disk stars of a given age in the solar neighborhood (Edvardsson et al., 1993; Reddy et al., 2003), metal abundance ratios (e.g., Mg/ $\mathrm{Fe}$ ) in the disk do not show resolvable scatter around the mean trends with metallicity. A number of heterogeneous GCE models have been published that attempt to explain abundance scatter (and its decrease with increasing metallicity) in the halo (Argast et al., 2000; Oey, 2000; Travaglio et al., 2001) and the large scatter of metallicity in the disk (e.g., Copi, 1997; van den Hoek and de Jong, 1997). We will consider the issue of heterogeneous GCE and its effects on presolar grain isotopic compositions in section 6.1.

In recent years, an additional class of GCE models has been explored, galactic chemodynamical (GCD) models, in which the chemical evolution of the galaxy is explicitly tied to its dynamical evolution. Galactic chemodynamical models range from relatively simple models exploring the effects of radial diffusion of stellar orbits coupled to abundance gradients (Grenon, 1987; François and Matteucci, 1993; Clayton, 1997) to quite sophisticated three-dimensional chemodynamic codes that attempt to self-consistently treat the dynamics of galactic gas, dust, and dark matter along with abundance evolution (Raiteri et al., 1996; Brook et al., 2003).

\section{NUCLEAR COSMOCHRONOLOGY AND EXTINCT RADIOACTIVITIES}

Nuclear cosmochronology, also sometimes termed nucleocosmochronology, is the study of radioactive nuclei with the goal of constraining the timescales of nucleosynthesis and galaxy formation. This field takes its root in a paper published in 1929, where E. Rutherford first used uranium to estimate the age of Earth and erroneously concluded that "the processes of production of elements like uranium were certainly taking place in the sun $4 \times 10^{9}$ years ago and probably still continue today" (Rutherford, 1929). Actually, the $\mathrm{U}$ in the solar system was produced, together with other actinides, by the $r$-process of nucleosynthesis in very energetic events, before the birth of the solar system (Meyer and Zinner, 2006). The primary aspiration of nuclear cosmochronology historically has been to retrieve the age of the Milky Way and the duration of nucleosynthesis from the abundances of unstable nuclei measured in meteorites.

The abundances of radioactive nuclides in the interstellar medium (ISM) represent a balance between production in stars and decay in the ISM (Tinsley, 1977, 1980; Yokoi et al., 1983; Clayton, 1985, 1988a; Pagel, 1997). These abundances depend very strongly on the dynamical evolution of the galaxy. For instance, the abundances of radioactivities in the ISM would be different if all nuclides had been synthesized in a stellar burst shortly after the formation of the galaxy or if they had been synthesized throughout the galactic history. Radioactivities therefore provide invaluable tools for probing the nucleosynthetic history of matter. In order to investigate the formation of the solar system, one can either proceed forward or backward in time. The abundances of radioactive nuclides in the ISM at solar system birth can be theoretically predicted from models of GCE and stellar nucleosynthesis. The abundances of radioactive nuclides can also be determined from laboratory measurements of extraterrestrial materials, for example, by detection of decay products of now-extinct nuclides. Comparisons between the predicted and the observed abundances provide unequaled pieces of information on galactic nucleosynthesis history and the origin of short-lived nuclides in the early solar system.

\subsection{Modeling the Remainder Ratio}

When investigating radionuclides in the ISM, it is useful to introduce the remainder ratio $(\mathfrak{R})$, which is the ratio of the abundance of an unstable nuclide to its abundance if it had been stable (Clayton, 1988a; Dauphas et al., 2003)

$$
\Re=\mathrm{N}(\tau) / \mathrm{N}(\tau \rightarrow \infty)
$$

If $\mathrm{R}$ is the ratio of the unstable nuclide to another stable nuclide cosynthesized at the same site and $\mathrm{P}$ is the production ratio, then the remainder ratio in the early solar system (ESS) can be calculated as

$$
\Re_{\text {ess }}=\mathrm{R} / \mathrm{P}
$$

The remainder ratio in the ISM at the time of solar system formation can be calculated in the framework of GCE models. Let us begin with the "simple" GCE model discussed in section 3. (Fig. 1). The remainder ratio in the ISM (equation (10)) can be calculated from equation (7)

$$
\Re_{\text {ism }}=\frac{\tau}{\mathrm{T}}\left(1-\mathrm{e}^{-\mathrm{T} / \tau}\right)
$$

The presolar age of the galaxy is denoted $\mathrm{T}\left(\mathrm{T}=\mathrm{T}_{\mathrm{G}}-\mathrm{T}_{\odot}\right.$, where $\mathrm{T}_{\mathrm{G}}$ is the age of the galaxy and $\mathrm{T}_{\odot}$ is the age of the solar system). Thus, in the closed-box linear model, the remainder ratio of a given nuclide depends on its mean-life $\tau$ and on the presolar age of the galactic disk T. For shortlived nuclides $(\tau \ll \mathrm{T})$, the remainder ratio becomes

$$
\Re_{\mathrm{ism}}=\tau / \mathrm{T}
$$

For very short-lived species, the timescale of ISM mixing is longer than the mean-life of the nuclide, granularity of nucleosynthesis must be taken into account, and the notion of steady-state abundances is inappropriate. Meyer and Clayton (2000) estimated that the cut where the concept of steady-state abundances breaks down must be for meanlives around 5 m.y. As discussed earlier, the simple model fails to explain important astronomical observations, notably the G-dwarf metallicity distribution. Clayton (1985, 1988a) estimated the remainder ratio for short-lived nuclides in the context of a parameterized linear infall model. More 
recently, Dauphas et al. (2003) improved over this model using a more realistic nonlinear parameterization of the star formation rate $\left[\mathrm{d} \sigma_{\mathrm{g}} / \mathrm{dt}=-\omega \sigma_{\mathrm{g}}^{\mathrm{n}}\right.$, with $\mathrm{n}$ close to 1.4 (Gerritsen and Icke, 1997; Kennicutt, 1998; Kravtsov, 2003)] and a parameterized infall rate following Chang et al. (1999). As discussed by Clayton (1988a) and Dauphas et al. (2003), when infall of low-metallicity gas is taken into account, equation (13) for the remainder ratio for short-lived nuclides is not valid and the expression

$$
\Re_{\mathrm{ISM}}=\kappa \tau / \mathrm{T}
$$

should be used instead. The numerical GCE model of Dauphas et al. (2003) gives $\kappa=2.7 \pm 0.4$ (unless otherwise indicated, all errors in this chapter are $2 \sigma$ ), which is within the range of values advocated by Clayton (1985), $2<\kappa<4$. For long-lived radionuclides, Clayton (1988a) derived an analytic solution for the remainder ratio. If nonlinearity is taken into account, the remainder ratio must be calculated numerically (equation (4) of Dauphas et al., 2003).

\subsection{Age of the Galaxy and the Uranium/Thorium Production Ratio}

As illustrated in the previous section, the remainder ratio in the ISM at the time of solar system formation depends on the age of the galactic disk. For short-lived radionuclides, it is possible that significant decay can occur between the last nucleosynthesis event and actual incorporation into the solar system's parent molecular cloud core (see section 4.3). For long-lived radionuclides, however, such a free-decay interval can be neglected and the remainder ratio in the ISM must be equal to that in the ESS. It is thus possible to determine the age of the galaxy if a GCE model is specified, if the abundance of the considered radionuclide in the ESS is known, and if its production ratio normalized to a neighbor nuclide co-synthesized at the same site is known. The first meaningful attempt to calculate the radiometric age of the Milky Way was reported in the seminal paper of Burbidge et al. (1957). Using a U/Th production ratio of 0.64 and a model of constant production, they estimated an age of approximately 10 G.y. for the galaxy. In the last 50 years, multiple studies have addressed this question and the reader will find ample details in some of these contributions (e.g., Tinsley, 1980; Yokoi et al., 1983; Clayton, 1988a; Cowan et al., 1991; Meyer and Truran, 2000). Clayton (1988a) evaluated potential nuclear cosmochronometers and concluded that the pair ${ }^{238} \mathrm{U}\left(\tau=6.446 \times 10^{9} \mathrm{yr}\right)-232 \mathrm{Th}(\tau=2.027 \times$ $10^{10} \mathrm{yr}$ ) gives the most stringent constraint on the age of the galactic disk. We shall therefore focus our discussion on the ${ }^{238} \mathrm{U} / 232 \mathrm{Th}$ ratio, which will simply be denoted U/Th hereafter. There are two approaches that can be used to estimate the age of the Milky Way based on the U/Th ratio. One relies on the determination of this ratio in the spectra of lowmetallicity stars in the halo of the galaxy. The second relies on the U/Th ratio measured in meteorites and makes use of galactic chemical evolution models.
The U/Th ratio measured in meteorites (Chen et al., 1993; Goreva and Burnett, 2001) is the result of an interplay between production in stars, enrichment of the gas by stellar ejecta, and decay in the ISM. Because U decays faster than Th, its ratio in the ISM changes with time. If one specifies the history of nucleosynthesis before solar system formation, a relationship can be found between the U/Th production ratio, the ratio measured in the ISM at solar system formation (as recorded in meteorites), and the age of the galaxy. For instance, let us consider that actinides were all synthesized at the time the galaxy formed and that they were not subsequently replenished (initial stellar burst scenario). In this case, we can write a simple free-decay equation

$$
\mathrm{R}_{\odot}^{\mathrm{U} / \mathrm{Th}}=\mathrm{P}^{\mathrm{U} / \mathrm{Th}} \mathrm{e}^{\left(\lambda_{\mathrm{Th}}-\lambda_{\mathrm{U}}\right)\left(\mathrm{T}_{\mathrm{G}}-\mathrm{T}_{\odot}\right)}
$$

where $\mathrm{R}_{\odot}^{\mathrm{U} / \mathrm{Th}}$ is the ratio in the ISM at solar system formation, $\mathrm{P}^{\mathrm{U} / \mathrm{Th}}$ is the production ratio, and $\mathrm{T}_{\mathrm{G}}$ is the total age of the galaxy (to present). The ratio in the ISM $\left(\mathrm{R}_{\odot}^{\mathrm{U} / \mathrm{Th}}\right)$ is measured in meteorites; the production ratio $(\mathrm{PU} / \mathrm{Th})$ can be derived from the theory of $r$-process nucleosynthesis. The age $\left(\mathrm{T}_{\mathrm{G}}\right)$ can therefore be calculated. Of course, all actinides were not produced in an initial burst and it is thus necessary to consider more realistic GCE models. Such models, describing enrichment of the ISM in actinides through time, can be constrained by a host of astronomical observations (Yokoi et al., 1983; Clayton, 1988a; Dauphas, 2005a), but a detailed discussion of how the models are parameterized and constrained is beyond the scope of this chapter. The most important feature of the models is that they incorporate infall of low-metallicity gas on the galactic plane. In a recent paper, Dauphas (2005a) showed that the relationship between the production ratio, the meteorite ratio, and the age of the galaxy can be approximated by a simple formula, valid between 10 and 20 G.y.

$$
\mathrm{P}^{\mathrm{U} / \mathrm{Th}}=\mathrm{R}_{\odot}^{\mathrm{U} / \mathrm{Th}} /\left(\mathrm{aT} \mathrm{T}_{\mathrm{G}}+\mathrm{b}\right)
$$

where $\mathrm{a}=-1.576 \times 10^{-2}$ and $\mathrm{b}=0.9946$ (see Dauphas, $2005 \mathrm{a}$, for details). The U/Th ratio in the ISM at solar system formation is $0.438 \pm 0.006$ (Chen et al., 1993). Goriely and Arnould (2001) and Schatz et al. (2002) recently quantified the influence of nuclear model uncertainties on the $r$-process nucleosynthesis of actinides. The ${ }^{238} \mathrm{U} / 232 \mathrm{Th}$ production ratio is estimated by Schatz et al. (2002) to be $0.60 \pm 0.14$, while Goriely and Arnould (2001) propose a more conservative range of $0.435_{-0.137}^{+0.329}$ (error bars represent $68 \%$ confidence intervals). The range of production ratios estimated by modern $r$-process calculations encompasses the initial solar composition and the approach based on GCE can therefore only provide an upper limit on the age of the galaxy. If we adopt $\mathrm{P}^{\mathrm{U} / \mathrm{Th}}<0.7$, we can derive an upper limit for the age of the Milky Way of approximately 20 G.y., which is useless in comparison to the precision with which the age of the universe is known [13.7 G.y. (Spergel et al., 2003)]. This shows that the solar U/Th ratio alone cannot be used to constrain the duration of nucleosynthesis. 
The U/Th ratio measured in low-metallicity halo stars can also be used as a potential chronometer to determine the age of the Milky Way (Cayrel et al., 2001; Hill et al., 2002; Cowan et al., 2002). These stars formed very early in the galactic history and they inherited at their formation a U/Th ratio that must have been equal to the production ratio by $r$-process nucleosynthesis. Hill et al. (2002) measured the most precise U/Th ratio in the low-metallicity halo star CS 31082-001 of 0.115 \pm 0.029 . For such stars, a simple free decay equation can be written

$$
\mathrm{P}^{\mathrm{U} / \mathrm{Th}}=\mathrm{R}_{\mathrm{LMHS}}^{\mathrm{U} / \mathrm{Th}} \mathrm{e}^{\left(\lambda_{\mathrm{U}}-\lambda_{\mathrm{Th}}\right) \mathrm{T}_{\mathrm{G}}}
$$

Again, the ratio $\mathrm{R}_{\mathrm{LMHS}}^{\mathrm{U} / \mathrm{Th}}$ in low-metallicity halo stars can be measured, the production ratio $\mathrm{P}^{\mathrm{U} / \mathrm{Th}}$ can be derived from the theory of $r$-process nucleosynthesis, and it is therefore possible to calculate the age of the galaxy $\mathrm{T}_{\mathrm{G}}$. Goriely and Arnould (2001) propagated the uncertainty on the production ratio and concluded that the age cannot be constrained to better than 9-18 G.y. As in the case of GCE and the solar $\mathrm{U} / \mathrm{Th}$ ratio, this range is of limited use when trying to establish the chronology of structure of formation in the universe and other methods give more precise estimate of the age of the galaxy (Krauss and Chaboyer, 2003; Hansen et al., 2004).

The main source of uncertainty in calculations of the age of the Milky Way based on low-metallicity halo stars and galactic chemical evolution is the $\mathrm{U} / \mathrm{Th}$ production ratio. In a recent contribution, Dauphas (2005a) argued that because

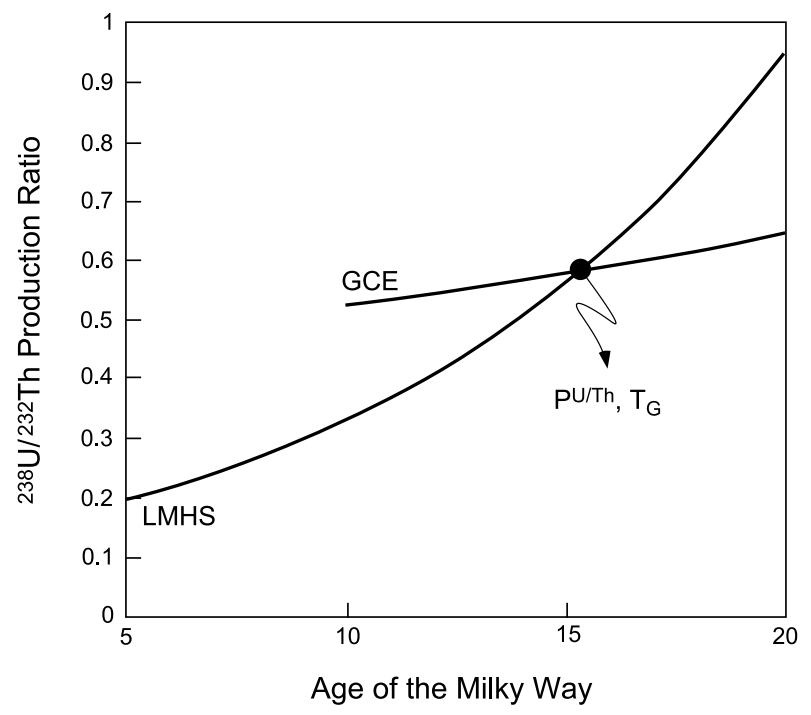

Fig. 3. Calculated ${ }^{238} \mathrm{U} / 232 \mathrm{Th}$ production ratio as a function of the age of the Milky Way based on the U/Th ratio measured in low-metallicity halo stars (curve LMHS, equation (17)) and the U/Th ratio measured in solar system material (curve GCE, equation (16)). By combining these two approaches, it is possible to estimate both the age $\mathrm{T}_{\mathrm{G}}$ and the production ratio $\mathrm{P}^{\mathrm{U} / \mathrm{Th}}$ (Dauphas, 2005a). there are two equations (equations (16) and (17)) in two unknowns $\left(\mathrm{P}^{\mathrm{U} / \mathrm{Th}}\right.$ and $\mathrm{T}_{\mathrm{G}}$ ), the system could actually be solved (Fig. 3). The values that he derived for the age and the production ratio are $14.5_{-2.2}^{+2.8}$ G.y. and $0.571_{-0.031}^{+0.037}$, respectively. The virtue of this approach is that a probabilistic meaning can be ascribed to the uncertainty interval and the U/Th production ratio can be determined independently of $r$-process calculations. The oldest stars in our galaxy formed shortly after the birth of the universe [13.7 \pm 0.2 G.y. (Spergel et al., 2003)].

\subsection{Short-Lived Nuclides in the Early Solar System}

The GCE models presented previously all assume that nucleosynthesis is a smooth function of time. Stars are actually discrete in both time and space. Long-lived radionuclides retain a long memory and deterministic models can be applied (Clayton, 1988a; Dauphas et al., 2003). In contrast, short-lived radionuclides may be affected by the nucleosynthetic history of the solar neighborhood right before solar system formation. For these nuclides, a stochastic treatment should be applied (e.g., Meyer and Luo, 1997). Very short-lived extinct radionuclides, such as ${ }^{26} \mathrm{Al}$, might have been injected from a nearby giant star [asymptotic giant branch (AGB) or supernova (SN)] that might have triggered the protosolar nebula into collapse. In the present contribution, the discussion is limited to extinct radionuclides that have mean-lives long enough that the composition of the ISM can be estimated in a deterministic way [ $\tau>5$ m.y. (Meyer and Clayton, 2000)]. This comprises the nuclides ${ }^{53} \mathrm{Mn}$ (mean-life 5.4 m.y.), ${ }^{92} \mathrm{Nb}$ (50.1 m.y.), ${ }^{107} \mathrm{Pd}$ (9.4 m.y.), ${ }^{129} \mathrm{I}$ (22.6 m.y.), ${ }^{146} \mathrm{Sm}$ (148.6 m.y.), ${ }^{182} \mathrm{Hf}$ (13.0 m.y.), and ${ }^{244} \mathrm{Pu}$ (115.4 m.y.). The abundances of all these nuclides in the ESS are well known. For calculating the remainder ratio, the abundances of these nuclides must be normalized to the abundances of neighbor nuclides produced in the same stellar environment. Hence, the normalizing nuclides used in the present contribution are not always identical to those used in the initial publications reporting extinct nuclide abundances. For evaluating the remainder ratio in the ESS, the basic ingredients are the normalized abundances of the short-lived nuclides in the ESS at the time of calcium-aluminum-rich inclusion (CAI) formation and the associated production ratios.

Manganese-53 is synthesized together with ${ }^{55} \mathrm{Mn}$ in massive stars. As discussed by Meyer and Clayton (2000), because solar system ${ }^{53} \mathrm{Cr}$ must have been primarily synthesized as ${ }^{53} \mathrm{Mn}$, the production ratio ${ }^{53} \mathrm{Mn} /{ }^{55} \mathrm{Mn}$ can be approximated by the solar system ${ }^{53} \mathrm{Cr} / 55 \mathrm{Mn}$ ratio of 0.13 (Anders and Grevesse, 1989). The SN II models of Rauscher et al. (2002) predict a comparable production ratio, ${ }^{53} \mathrm{Mn} /$ ${ }^{55} \mathrm{Mn}=0.15$, when individual yields from SN II of different masses are weighted by a typical initial mass function. Note that Sne II underproduce Fe-peak nuclides relative to ${ }^{16} \mathrm{O}$ by a factor of 2-3 (Rauscher et al., 2002); the rest must be produced in SN Ia. This underproduction feature is reflected in 
the abundance patterns of low-metallicity halo stars (Wheeler et al., 1989; McWilliam, 1997). The initial ${ }^{53} \mathrm{Mn} / 55 \mathrm{Mn}$ ratio in CAIs is estimated to be $2.81 \pm 0.31 \times 10^{-5}$ (Birck and Allègre, 1985; Nyquist et al., 2001). However, a lower initial value may be required in order to bring the chronologies based on the various extinct and extant radionuclides into agreement (Lugmair and Shukolyukov, 1998; Dauphas et al., 2005). We shall adopt here an initial ratio of $1.0 \pm 0.2 \times$ $10^{-5}$. The remainder ratio is therefore $\mathfrak{R}_{\mathrm{ess}}^{53}=7.7 \pm 1.5 \times 10^{-5}$.

Niobium-92 was most likely synthesized by the $p$-process in SN. This radionuclide cannot be normalized to another isotope of the same element because the only stable isotope of $\mathrm{Nb},{ }^{93} \mathrm{Nb}$, was synthesized by the $s$-process. It can instead be normalized to ${ }^{92} \mathrm{Mo}$, which is also a pure $p$-process nuclide. The ${ }^{92} \mathrm{Nb} /{ }^{92}$ Mo production ratio during photodisintegration of seed nuclei in SN II is $1.5 \pm 0.6 \times 10^{-3}$ (Rauscher et al., 2002; Dauphas et al., 2003). The initial ratio in meteorites is $2.8 \pm 0.5 \times 10^{-5}$ (Harper, 1996; Schönbächler et al., 2002). The remainder ratio is therefore $\mathfrak{R}_{\mathrm{ess}}^{92}=1.9 \pm 0.8 \times$ 10-2 (Dauphas et al., 2003). Note that Münker et al. (2000) and Yin et al. (2000) found higher initial ratios, but the CAI measurements of Münker et al. (2000) might have been affected by nucleosynthetic effects, and the zircon measurement of Yin et al. (2000) was not replicated by Hirata (2001).

The initial ${ }^{107} \mathrm{Pd} /{ }^{110} \mathrm{Pd}$ in the ESS was $5.6 \pm 1.1 \times 10^{-5}$ (Kelley and Wasserburg, 1978; Chen and Wasserburg, 1996). This corresponds to ${ }^{107} \mathrm{Pd} /{ }^{10} \mathrm{Pd}^{\mathrm{r}}=5.8 \pm 1.2 \times 10^{-5}$, where ${ }^{110} \mathrm{Pd}^{\mathrm{r}}$ is the $r$-process contribution to the solar abundance of ${ }^{110} \mathrm{Pd}$ (Arlandini et al., 1999). Palladium-107 is primarily a $r$-process nuclide that could also have received a contribution from the $s$-process. For most $r$-process radionuclides, their production ratios can be reliably estimated by decomposing the abundances of their daughter nuclides into $s$ - and $r$-process contributions. For instance, the entire $r$-process abundance of ${ }^{107} \mathrm{Ag}$ must have been channeled through ${ }^{107} \mathrm{Pd}$. The sites of $r$-nucleosynthesis are not well established but they very likely correspond to the late stages of rapidly evolving stars. The ${ }^{107} \mathrm{Pd} / 110 \mathrm{Pd}$ production ratio can therefore be approximated to the solar system ${ }^{107} \mathrm{Agr} / 1{ }^{10} \mathrm{Pdr}$, where ${ }^{107} \mathrm{Ag}^{\mathrm{r}}$ and ${ }^{110} \mathrm{Pd}^{\mathrm{r}}$ are obtained by subtracting the $s$-process contribution to solar abundances (Arlandini et al., 1999). The ${ }^{107} \mathrm{Pd} / 110 \mathrm{Pd} r$-process production ratio is therefore 1.36 and the remainder ratio is $\Re_{\mathrm{ess}}^{107}=4.3 \pm 0.9 \times 10^{-5}$.

Iodine-129 is also primarily an $r$-process nuclide. It was historically the first short-lived nuclide to have been found to have been alive in the ESS (Reynolds, 1960). When the $\mathrm{Pb} / \mathrm{Pb}$ age of Efremovka CAIs [4567.2 $\pm 0.6 \mathrm{Ma}$ (Amelin et al., 2002)] is combined with the observed ${ }^{129} \mathrm{I} /{ }^{127} \mathrm{I}-\mathrm{Pb} /$ $\mathrm{Pb}$ age correlation in ordinary chondrite phosphates (Brazzle et al., 1999), the early solar system ${ }^{129} \mathrm{I} /{ }^{127} \mathrm{I}$ is estimated to be $1.19 \pm 0.20 \times 10^{-4}$. This corresponds to a ${ }^{129} \mathrm{I} /{ }^{127} \mathrm{Ir}$ initial ratio of $1.25 \pm 0.21 \times 10^{-4}$. As discussed in the case of ${ }^{107} \mathrm{Pd}$, the ${ }^{129} \mathrm{I} /{ }^{127} \mathrm{I} r$-process production ratio can be estimated from decomposition of ${ }^{129} \mathrm{Xe}$ into $r$ - and $s$-processes (Arlandini et al., 1999). Because ${ }^{129} \mathrm{Xe}$ is predominantly synthesized by the $r$-process, little uncertainty affects the production ratio, ${ }^{129} \mathrm{I} / 127 \mathrm{I}=1.45$. The remainder ratio for ${ }^{129} \mathrm{I}$ is $\mathfrak{R}_{\mathrm{ess}}^{129}=$ $8.6 \pm 1.5 \times 10^{-5}$.

Samarium-146 is a pure $p$-process isotope. The ESS ${ }^{146} \mathrm{Sm} /{ }^{144} \mathrm{Sm}$ ratio is $7.6 \pm 1.3 \times 10^{-3}$ (Lugmair et al., 1983 ; Prinzhofer et al., 1992). Its production ratio as obtained in the most recent models of the $p$ - or $\gamma$-process in Sne II is $1.8 \pm 0.6 \times 10^{-1}$ (Rauscher et al., 2002; Dauphas et al., 2003). The remainder ratio is therefore $\mathfrak{R}_{\mathrm{ess}}^{146}=4.2 \pm 1.6 \times$ 10-2 (Dauphas et al., 2003).

Hafnium-182 is presumably an $r$-process isotope. From the decomposition of the abundance of its daughter isotope 182W into $r$ - and $s$-processes (Arlandini et al., 1999), the ${ }^{182} \mathrm{Hf} / 177 \mathrm{Hf}$ production ratio is estimated to be 0.81 . The initial ${ }^{182} \mathrm{Hf} /{ }^{177} \mathrm{Hf}$ ratio in the ESS is $1.89 \pm 0.15 \times 10^{-4}$ (Yin et al., 2002). Note that Quitté and Birck (2004) have derived a higher initial ratio from $\mathrm{W}$-isotopic measurements of the Tlacotepec iron meteorite, but this may be affected by cosmogenic effects. The initial ratio of Yin et al. (2002) corresponds to ${ }^{182} \mathrm{Hf} /{ }^{177} \mathrm{Hfr}=2.31 \pm 0.18 \times 10^{-4}$. The remainder ratio is therefore $\Re^{182}=2.86 \pm 0.23 \times 10^{-4}$.

Plutonium-244 is an $r$-process isotope. As with other actinides, its production ratio is uncertain because the closest stable $r$-process nuclide that can anchor the models is ${ }^{209} \mathrm{Bi}$, 35 amu away. Goriely and Arnould (2001) evaluated nuclear model uncertainties on the production of actinides. Among the various models, only those that give a ${ }^{238 \mathrm{U} / 232 \mathrm{Th} \text { pro- }}$ duction ratio consistent with meteoritic abundances are retained. The ${ }^{244} \mathrm{Pu} /{ }^{238} \mathrm{U}$ production ratio is thus estimated to be $0.53 \pm 0.36$. The initial ${ }^{244} \mathrm{Pu} /{ }^{238} \mathrm{U}$ in the solar system is estimated to be $0.0068 \pm 0.0010$ (Rowe and Kuroda, 1965; Hudson et al., 1989). The ratio of the remainder ratios $\mathfrak{R}_{\mathrm{ess}}^{244} / \mathfrak{R}_{\mathrm{ess}}^{238}$ is therefore $1.28 \pm 0.89 \times 10^{-2}$. The remainder ratio of $238 \mathrm{U}$ can be estimated in the framework of the open nonlinear GCE model (Dauphas et al., 2003) to be 0.71 for a galactic age of 13.7 G.y. (0.53 in the closed-box model). The remainder ratio of ${ }^{244} \mathrm{Pu}$ is thus $\mathfrak{R}_{\text {ess }}^{244}=9.1 \pm 6.3 \times 10^{-3}$ (Dauphas, 2005b). All these ratios are compiled in Table 1.

The remainder ratios in the ESS can be compared with those in the ISM as predicted by GCE models. In order to account for the possible isolation of the solar system parent molecular cloud core from fresh nucleosynthetic inputs, a free-decay interval $(\Delta)$ is often introduced [see Clayton (1983) for a more complicated treatment]. This corresponds to a time when radioactive species decay without being replenished by stellar sources. The remainder ratio in the ESS is related to that in the ISM through

$$
\mathfrak{R}_{\mathrm{ess}}=\mathfrak{R}_{\mathrm{ism}} \exp \left(-\frac{\Delta}{\tau}\right)
$$

The remainder ratio in the ISM is itself a function of the mean-life of the considered nuclide (equation (14)). For radionuclides whose mean-lives are long enough that their abundances in the ESS can be explained by their steadystate abundance in the ISM, combining equations (14) and 
TABLE 1. Extinct radionuclides in the ESS (only those with mean-lives $\tau>5$ m.y. are listed, Dauphas et al., 2003; Dauphas, 2005b).

\begin{tabular}{lcccccc}
\hline Nuclide & $\tau(\mathrm{Ma})$ & Norm. & $\mathrm{R}$ & $\mathrm{P}$ & $\Re_{\text {ess }}^{\mathrm{i}}$ & $\mathfrak{R}_{\mathrm{ism}}^{\mathrm{i}}$ \\
\hline Manganese-53 & 5.4 & $55 \mathrm{Mn}$ & $1.0 \pm 0.2 \times 10^{-5}$ & $\sim 1.3 \times 10^{-1}$ & $7.7 \pm 1.5 \times 10^{-5}$ & $1.67 \pm 0.38 \times 10^{-3}$ \\
Niobium-92 & 50.1 & $92 \mathrm{Mo}$ & $2.8 \pm 0.5 \times 10^{-5}$ & $1.5 \pm 0.6 \times 10^{-3}$ & $1.9 \pm 0.8 \times 10^{-2}$ & $1.55 \pm 0.35 \times 10^{-2}$ \\
Palladium-107 & 9.4 & $110 \mathrm{Pd}^{\mathrm{r}}$ & $5.8 \pm 1.2 \times 10^{-5}$ & $\sim 1.36$ & $4.3 \pm 0.9 \times 10^{-5}$ & $2.91 \pm 0.66 \times 10^{-3}$ \\
Iodine-129 & 22.6 & $127 \mathrm{I}$ & $1.25 \pm 0.21 \times 10^{-4}$ & $\sim 1.45$ & $8.6 \pm 1.5 \times 10^{-5}$ & $7.03 \pm 1.6 \times 10^{-3}$ \\
Samarium-146 & 148.6 & ${ }^{144} \mathrm{Sm}$ & $7.6 \pm 1.3 \times 10^{-3}$ & $1.8 \pm 0.6 \times 10^{-1}$ & $4.2 \pm 1.6 \times 10^{-2}$ & $4.61 \pm 1.05 \times 10^{-2}$ \\
Hafnium-182 & 13.0 & ${ }^{177} \mathrm{Hf} \mathrm{r}$ & $2.31 \pm 0.18 \times 10^{-4}$ & $\sim 0.81$ & $2.86 \pm 0.23 \times 10^{-4}$ & $4.03 \pm 0.92 \times 10^{-3}$ \\
Plutonium-244 & 115.4 & ${ }^{238} \mathrm{U}$ & $6.8 \pm 1.0 \times 10^{-3}$ & $5.3 \pm 3.6 \times 10^{-1}$ & $9.1 \pm 6.3 \times 10^{-3}$ & $3.58 \pm 0.81 \times 10^{-2}$ \\
\hline
\end{tabular}

The superscript $\mathrm{r}$ refers to the $r$-process component of the cosmic abundances [obtained after subtracting the $s$-process contribution (Arlandini et al., 1999)]. $\mathrm{R}$ is the ratio observed in the ESS, $\mathrm{P}$ is the production ratio, $\mathfrak{R}_{\mathrm{ess}}^{\mathrm{i}}$ is the remainder ratio in the ESS calculated as $\mathrm{R} / \mathrm{P}$ (except for ${ }^{244} \mathrm{Pu}$, for which the normalizing isotope is unstable and the remainder ratio must be corrected for $\mathfrak{R}^{238}=0.71$ ), $\Re_{\text {ism }}^{\mathrm{i}}$ is the remainder ratio in the ISM as obtained from an open nonlinear GCE model (Dauphas et al., 2003). The remainder ratio in the ISM is equal to $\Re_{\mathrm{ism}}^{\mathrm{i}}=\kappa \tau / \mathrm{T}$, with $\kappa=2.7 \pm 0.4$ and $\mathrm{T}=8.7 \pm 1.5 \mathrm{G}$.y. All errors are $2 \sigma$. See text for details and references.

(18) gives a relationship between the remainder ratios in the ISM and the ESS (Dauphas et al., 2003; Dauphas, 2005b)

$$
\Re_{\text {ess }}=\Re_{\text {ism }} \exp \left(-\frac{\kappa \Delta}{\mathrm{T} \Re_{\text {ism }}}\right)
$$

The remainder ratios of several short-lived radionuclides determined in the ESS are plotted vs. the ISM ratios derived from GCE models in Fig. 4. Also shown are curves corre-

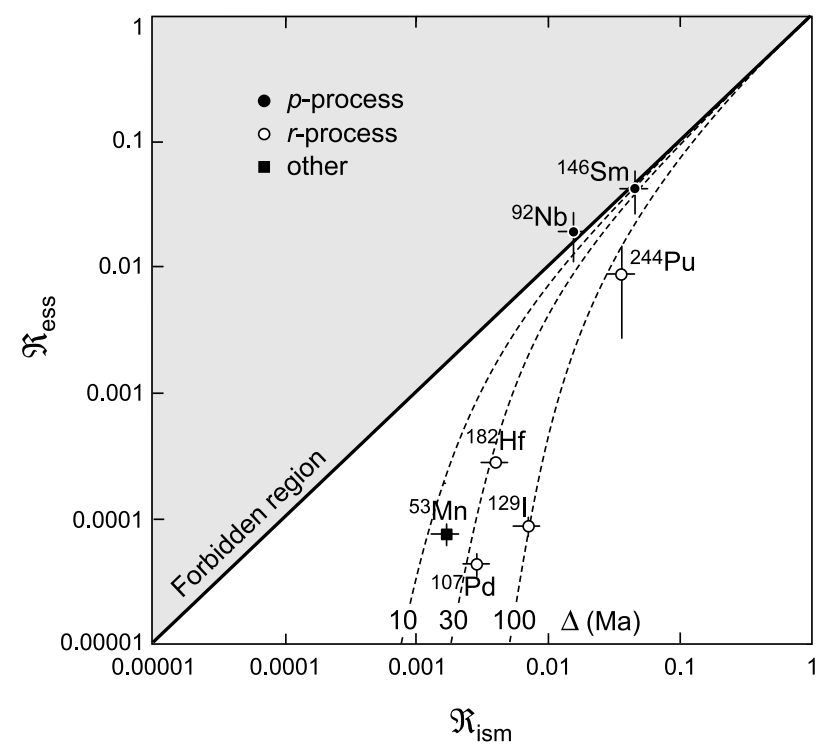

Fig. 4. Remainder ratios of several extinct nuclides in the ESS $\left(\Re_{\text {ess }}=\mathrm{R} / \mathrm{P}\right)$ are plotted against the corresponding remainder ratios in the ISM (see text). Theoretical expectations corresponding to different free-decay intervals $(\Delta)$ are represented as dashed-curves (equation (19)) (Dauphas et al., 2003; Dauphas, 2005b). Extinct radionuclides are grouped according to nucleosynthetic processes. The forbidden region is the place where $\mathfrak{R}_{\text {ess }}$ is higher than $\mathfrak{R}_{\text {ism }}$ (the extinct radionuclides cannot be inherited from GCE). Error bars are $2 \sigma$. sponding to equation (19) calculated with different values of the free-decay interval $\Delta$. Some implications of the presence of extinct radionuclides in meteorites on stellar nucleosynthesis and solar system formation are discussed in detail in the following sections.

4.3.1. Niobium-92 and the nucleosynthesis of molybdenum-ruthenium p-isotopes. The radionuclides ${ }^{53} \mathrm{Mn}$ and ${ }^{146} \mathrm{Sm}$ define the same free-decay interval within uncertainties $(\Delta \simeq 10 \mathrm{~m} . \mathrm{y}$.). They were probably synthesized in supernovae and were inherited in the ESS from GCE. Niobium-92 is a special case because it lies in a mass region of the nuclide chart where $\mathrm{SN}$ models underproduce $p$-process isotopes $\left({ }^{92} \mathrm{Mo},{ }^{94} \mathrm{Mo},{ }^{96} \mathrm{Ru}\right.$, and ${ }^{98} \mathrm{Ru}$ ) by a factor of 10 (Rauscher et al., 2002). This nuclide can therefore be used to test the various hypotheses that have been advanced to remedy the underproduction feature of supernovae in the Mo-Ru mass region (Yin et al., 2000; Dauphas et al., 2003).

4.3.2. The puzzling origins of extinct $r$-radioactivities. The $r$-process radionuclides ${ }^{129} \mathrm{I}$ and ${ }^{244} \mathrm{Pu}$ were inherited from GCE with a free-decay interval of approximately $100 \mathrm{~m}$.y. (Fig. 4). If the other $r$-process nuclides ${ }^{107} \mathrm{Pd}$ and ${ }^{182} \mathrm{Hf}$ had been inherited from GCE with the same freedecay interval (100 m.y.), then their abundances in the ESS would have been much lower than what is observed in meteorites (they require a shorter free-decay interval, 30 m.y.). The extinct radionuclides ${ }^{107} \mathrm{Pd}$ and ${ }^{182} \mathrm{Hf}$ must therefore have a different origin. Two distinct scenarios have been advocated for explaining the origin of these two short-lived nuclides.

Wasserburg et al. (1996) and Qian et al. (1998) questioned the universality of the so-called $r$-process. They suggested that two kinds of $r$-process events are responsible for the nucleosynthesis of neutron-rich nuclei. One of these events would synthesize heavy $r$-nuclei and actinides $\left({ }^{182} \mathrm{Hf}\right.$ and ${ }^{244} \mathrm{Pu}, \mathrm{H}$ events) while the other would synthesize light $r$-nuclei (129I, L events). The $\mathrm{H}$ events would occur 10 times more frequently than the L events, which would explain why the free-decay interval inferred from ${ }^{182} \mathrm{Hf}$ is lower than that inferred from ${ }^{129} \mathrm{I}$. Observations of elemental abundances 
in low-metallicity stars support the view that all $r$-nuclides are not produced at the same site (Sneden et al., 2000; Hill et al., 2002). Such stars formed early enough in the galactic history that contributions of a limited number of stars can be seen in their spectra. Sneden et al. (2000) analyzed the ultra-low-metallicity $([\mathrm{Fe} / \mathrm{H}]=-3.1)$ halo star CS 22892052 and found that low-mass $r$-process elements such as $\mathrm{Y}$ and Ag were deficient compared to expectations based on heavier $r$-process elements. More recently, Hill et al. (2002) determined the abundance of $\mathrm{U}, \mathrm{Th}$, and $\mathrm{Eu}$ in another metal-poor $([\mathrm{Fe} / \mathrm{H}]=-2.9)$ halo star $(\mathrm{CS} 31082-001)$. The age of this star based on the U/Th ratio is $14.5_{-2.2}^{+2.8}$ G.y. (section 4.2), which agrees with independent estimates of galactic ages. In contrast, the $\mathrm{Th} / \mathrm{Eu}$ ratio corresponds to an age that is younger than the age of the solar system, which is impossible (Hill et al., 2002). This suggests that actinides were produced independently of lighter $r$-process nuclides. Observations of low-metallicity stars therefore point to a multiplicity of $r$-process events, possibly as many as three. Wasserburg et al. (1996) and Qian et al. (1998) grouped ${ }^{182} \mathrm{Hf}$ with actinides, including ${ }^{244} \mathrm{Pu}$. However, Dauphas (2005b) showed that ${ }^{244} \mathrm{Pu}$ requires a longer free-decay interval ( $\Delta \simeq 100$ m.y.) compared to ${ }^{182} \mathrm{Hf}$ ( $\Delta \simeq 30$ m.y.). This discrepancy may indicate that, in addition to the $\mathrm{L}$ and $\mathrm{H}$ events, another event must be added to explain actinides (A events). Note that this would be consistent with observations of low-metallicity stars, which require three distinct production sites. According to the multiple $r$-processes model, ${ }^{107} \mathrm{Pd}$ was synthesized by the $s$-process in a lowmetallicity, high-mass AGB star that polluted the ESS with a stellar wind (Wasserburg et al., 1994; Gallino et al., 2004). Alternatively, it could have been injected by the explosion of a nearby SN, where it would have been synthesized by the weak $s$-process (Meyer and Clayton, 2000).

Another possible scenario is that the nuclides that are overabundant in the ESS compared to GCE expectations $\left({ }^{107} \mathrm{Pd}\right.$ and ${ }^{182} \mathrm{Hf}$, as well as ${ }^{26} \mathrm{Al},{ }^{36} \mathrm{Cl},{ }^{41} \mathrm{Ca}$, and $\left.{ }^{60} \mathrm{Fe}\right)$ were injected in the presolar molecular cloud core by the explosion of a nearby SN that might have triggered the protosolar cloud into collapse (Cameron and Truran, 1977; Meyer and Clayton, 2000; Meyer et al., 2004). The dynamical feasibility of injecting fresh nucleosynthetic products in the ESS has been studied carefully by Vanhala and Boss (2002). In the most recent version of the pollution model, it is assumed that only a fraction of the stellar ejecta is efficiently injected in the nascent solar system [the injection mass cut is the radius in mass coordinates above which the envelope of the $\mathrm{SN}$ is injected (Cameron et al., 1995; Meyer et al., 2004)]. For a $25 \mathrm{M}_{\odot}$ star, an injection mass cut of $5 \mathrm{M}_{\odot}$, and a time interval of $1 \mathrm{~m} . \mathrm{y}$. between the SN explosion and incorporation in the ESS, the abundances of ${ }^{26} \mathrm{Al},{ }^{41} \mathrm{Ca},{ }^{60} \mathrm{Fe}$, and ${ }^{182} \mathrm{Hf}$ are successfully reproduced (Meyer et al., 2004). Chlorine36 and ${ }^{107} \mathrm{Pd}$ are slightly overproduced but this may reflect uncertainties in ESS abundances and input physics. Because the injection scenario requires only one source for explaining all extinct radionuclides that cannot be produced by
GCE or irradiation in the ESS while the multiple $r$-processes scenario requires many, the principle of Ockham's Razor favors the SN pollution model.

4.3.3. Predicted abundance of curium-247 in the early solar system. Among the short-lived nuclides that have mean-lives higher than 5 m.y., only one has eluded detection, ${ }^{247} \mathrm{Cm}\left[\tau=22.5 \mathrm{~m}\right.$.y., decays to ${ }^{235 \mathrm{U}}$ (Chen and Wasserburg, 1981; Friedrich et al., 2004)]. Modeling of stellar nucleosynthesis and GCE allows the prediction of its abundance in the ESS. Curium-247 is expected to be produced at the same site that synthesized ${ }^{129} \mathrm{I}$ and ${ }^{244} \mathrm{Pu}$. The same free-decay interval can therefore be applied $(\Delta=100 \pm$ 25 m.y.). Goriely and Arnould (2001) estimated uncertainties on actinide production ratios. As already discussed in the case of ${ }^{244} \mathrm{Pu}$, we only consider the models that give a 238U/232Th production ratio consistent with the meteoritic measurement. The inferred ${ }^{247} \mathrm{Cm} / 238 \mathrm{U}$ production ratio is $0.138 \pm 0.054$. The remainder ratio in the $\mathrm{ISM}$ of ${ }^{247} \mathrm{Cm}$ can be calculated using the open nonlinear GCE model of Dauphas et al. $(2003), \mathfrak{R}_{\mathrm{ism}}^{247}=7.0 \pm 1.6 \times 10^{-3}$. Using the estimated remainder ratio of $238 \mathrm{U}(0.71$, see discussion on ${ }^{244} \mathrm{Pu}$ ), we get the ratio of the remainder ratios of ${ }^{247} \mathrm{Cm}$ and ${ }^{238} \mathrm{U}, \mathfrak{R}_{\mathrm{ism}}^{247} / \mathfrak{R}_{\mathrm{ism}}^{238}=9.8 \pm 2.2 \times 10^{-3}$. Allowing for free decay $\Delta=100 \pm 25$ m.y., the ratio in the ESS is $\mathfrak{R}_{\text {ess }}^{247} / \Re_{\text {ess }}^{238}=$ $1.2 \pm 0.4 \times 10^{-4}$. This value must be multiplied by the production ratio to get the expected ratio in meteorites. The ratio in the ESS is thus estimated to be ${ }^{247} \mathrm{Cm} / 238 \mathrm{U}=1.6 \pm$ $0.8 \times 10^{-5}\left({ }^{247} \mathrm{Cm} /{ }^{235} \mathrm{U}=5.0 \pm 2.6 \times 10^{-5}\right.$ at solar system formation). The present upper limit for the ${ }^{247} \mathrm{Cm} / 235 \mathrm{U}$ ratio obtained from U-isotopic measurements of meteoritic materials is $4 \times 10^{-3}$ (Chen and Wasserburg, 1981), which is entirely consistent with the predicted abundance based on modeling of GCE and nucleosynthesis.

\section{GALACTIC CHEMICAL EVOLUTION OF STABLE-ISOTOPE RATIOS}

As discussed above in section 2, elemental abundance ratios measured in stars are a powerful tool for constraining models of GCE, because different elements are made by different processes in different types of stars with different evolutionary timescales. This statement is of course not limited to elements, but applies equally well to stable-isotope ratios. Many elements are comprised of both primary and secondary isotopes, as defined in section 2, so GCE theory anticipates that many isotopic ratios should evolve in the galaxy. As discussed above, the simple closed-box GCE model predicts that the ratio of a secondary to a primary isotope increases linearly with metallicity in the galaxy. As an example, let us consider the stable isotopes of silicon. Numerical supernova nucleosynthetic calculations (Woosley and Weaver, 1995; Timmes and Clayton, 1996) indicate that ${ }^{28} \mathrm{Si}$ is a primary isotope, whereas ${ }^{29} \mathrm{Si}$ and ${ }^{30} \mathrm{Si}$ are secondary. Thus, it is not surprising that the numerical GCE model of Timmes and Clayton (1996) predicts that the ${ }^{29} \mathrm{Si} /{ }^{28} \mathrm{Si}$ and ${ }^{30} \mathrm{Si} / 28 \mathrm{Si}$ ratios increase monotonically with metallicity. The 


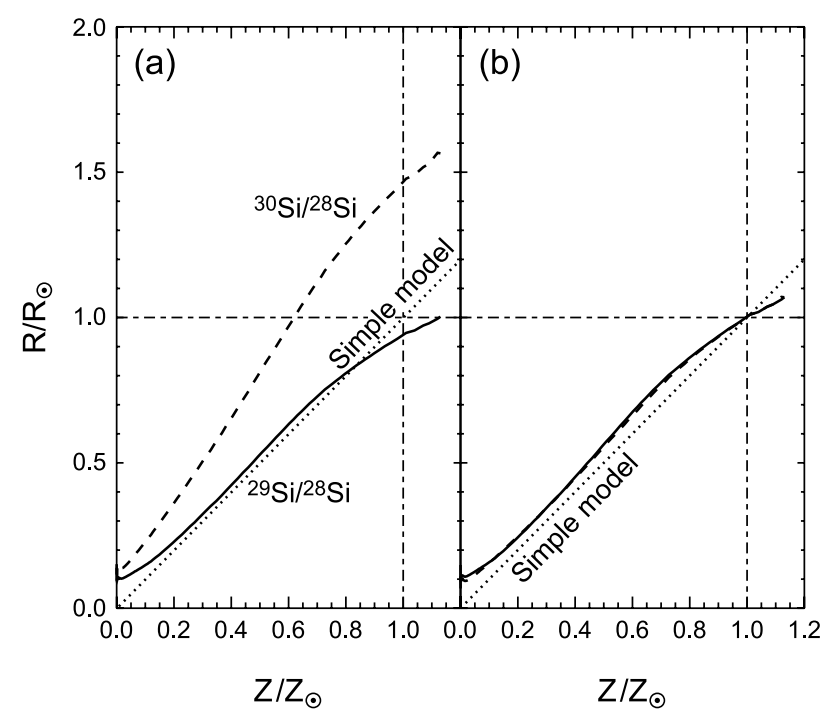

Fig. 5. Silicon-isotopic evolution predicted in the solar neighborhood by Timmes and Clayton (1996) and by the "simple" closedbox GCE model (R stands for isotopic ratio). Silicon-isotope ratios increase with metallicity, due to secondary nature of the heavy isotopes ${ }^{29} \mathrm{Si}$ and ${ }^{30} \mathrm{Si}$. (a) Unnormalized calculation: the predicted ratios miss the solar composition due to errors in nucleosynthesis models. (b) Isotopic ratios "renormalized" to reproduce the solar values at solar metallicity. The calculated isotope trends are shallower near solar metallicity than is predicted by the simple model of GCE.

$\mathrm{Si}$-isotopic ratios as a function of metallicity predicted by this model are shown in Fig. 5a. The secondary nature of the rare $\mathrm{Si}$ isotopes is clear. However, it is also immediately apparent that the model does not exactly reproduce the solar isotopic ratios at solar metallicity; the ${ }^{30} \mathrm{Si} / 28 \mathrm{Si}$ ratio is high by $\sim 50 \%$ and the ${ }^{29} \mathrm{Si} / 28 \mathrm{Si}$ ratio is slightly subsolar. The discrepancy is almost certainly due to errors in the supernova nucleosynthesis calculations that went into the GCE model, and agreement within a factor of 2 is usually considered a success in GCE modeling. However, this is not sufficient for comparison with presolar grain data measured with $1 \%$ precision (see next section). As discussed at length by Timmes and Clayton (1996), to compare high-precision isotope data with the GCE models in a self-consistent way requires that the models be "renormalized" so that they reproduce the solar abundances. The simplest approach is to rescale the GCE trends so that they pass through the solar composition at solar metallicity. The renormalized Si-isotope trends, scaled in this fashion, are shown in Fig. 5b. With this renormalization, it is clear that the GCE model predicts that the ratios vary in lockstep with one another during galactic evolution. This reflects that ${ }^{29} \mathrm{Si}$ and ${ }^{30} \mathrm{Si}$ are made in similar processes in supernovae. The dotted line indicates the prediction of the simple model for a pure secondary/primary ratio. The renormalized numerical trends clearly show a shallower metallicity dependence near solar metallicity than is predicted by the simple model.
An early application of the idea that isotope ratios evolve in the galaxy was proposed by Clayton (1988b) to explain the well-known ${ }^{16} \mathrm{O}$ excess measured in CAIs in meteorites (Clayton et al., 1973; Clayton, 1993). In this model, the meteoritic data represents a "chemical memory" of interstellar dust. The interstellar dust is postulated to consist of refractory cores of $\mathrm{Al}_{2} \mathrm{O}_{3}$ mantled by more volatile $\mathrm{O}$-bearing materials. Because the cores are more robust to destructive processes, they are on average older than the dust mantles. Analogous to $\mathrm{Si},{ }^{17} \mathrm{O} /{ }^{16} \mathrm{O}$ and ${ }^{18} \mathrm{O} /{ }^{16} \mathrm{O}$ ratios are expected to increase in the galaxy with time, since ${ }^{16} \mathrm{O}$ is a primary isotope and the others are secondary. Thus, the older refractory dust cores are expected to be rich in ${ }^{16} \mathrm{O}$, relative to the bulk ISM, which is dominated by more recent stellar ejecta. If the CAIs formed from materials preferentially enriched in the refractory interstellar dust cores, their ${ }^{16} \mathrm{O}$-richness, compared to typical solar system materials, could be naturally explained. Current belief favors chemical processes in the early solar system to explain the CAI O-isotope data (e.g., Thiemens and Heidenreich, 1983; Clayton, 2002), but the GCE suggestion of Clayton (1988b) has never been disproved.

\section{PRESOLAR GRAINS IN METEORITES AND GALACTIC CHEMICAL EVOLUTION}

Presolar grains are micrometer-sized and smaller solid samples of stars that can be studied in the laboratory (Bernatowicz et al., 1987; Lewis et al., 1987; Zinner, 1998; Nittler, 2003; Clayton and Nittler, 2004; Meyer and Zinner, 2006). They formed in stellar outflows more than 4.6 G.y. ago, became part of the Sun's parent molecular cloud, survived formation of the solar system, and became trapped in asteroids and comets, samples of which now intersect Earth as meteorites and interplanetary dust. They are recognized as presolar grains by their extremely unusual isotopic compositions. These compositions reflect those of the gas from which they condensed and thus provide a great deal of information about the nuclear history of their parent stars. Two types of presolar grains are believed to provide information about GCE (in addition to information about the evolutionary processes of the individual parent stars): $\mathrm{SiC}$ and oxides, mostly $\mathrm{Al}_{2} \mathrm{O}_{3}$ and $\mathrm{MgAl}_{2} \mathrm{O}_{4}$.

As discussed in the previous section, many isotopic ratios are expected to evolve in the galaxy. Because the isotopic ratios of individual presolar grains can in many cases be measured with much higher precision than element ratios can be measured in stars, these grains have the potential to provide additional constraints on GCE. Note, however, that the grains formed in disk stars, probably between $\sim 5$ and 10 G.y. ago, and thus span a narrower range of time than can be probed by astronomical observations. Moreover, their parent stars have been dead for eons and thus their properties (stellar type, mass, etc.) have to be inferred from the grain properties, primarily isotopic compositions, themselves. 

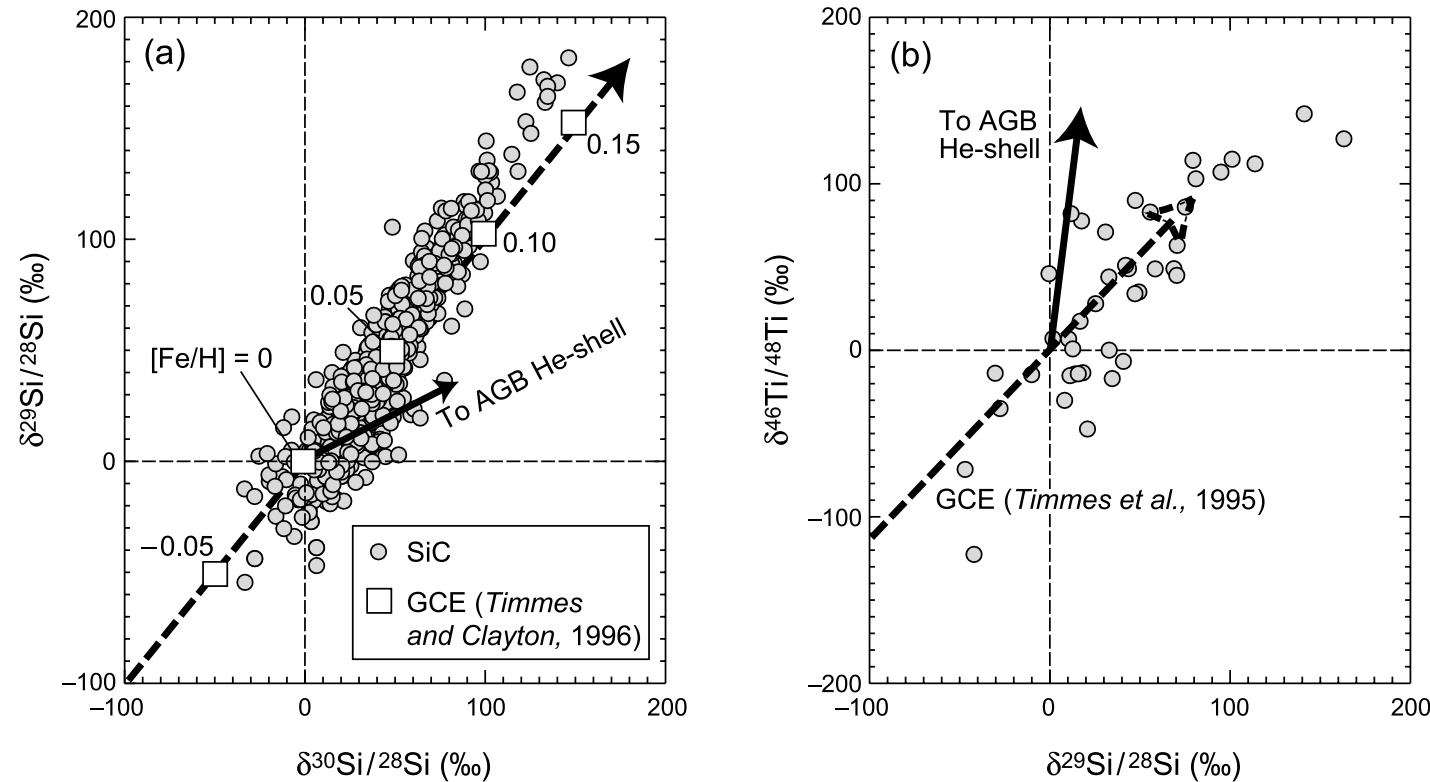

Fig. 6. Silicon- and Ti-isotopic ratios of mainstream presolar SiC grains (Hoppe et al., 1994; Alexander and Nittler, 1999). Ratios are expressed as $\delta$ values, permil deviations from a terrestrial isotope standard: $\delta \mathrm{R}=\left[\mathrm{R}_{\text {meas }} / \mathrm{R}_{\text {standard }}-1\right] \times 10^{3}$. The grain data are correlated in both plots, but the slopes are different from expectations for dredge-up in single AGB stars (Lugaro et al., 1999). Also shown are renormalized GCE calculations (see Fig. 5) of isotopic evolution (Timmes and Clayton, 1996; Timmes et al., 1995); metallicity values are indicated in (a).

\subsection{Galactic Chemical Evolution and Presolar Silicon Carbide}

The best-studied presolar phase in meteorites is silicon carbide ( $\mathrm{SiC})$. The vast majority of these grains (the "mainstream" population) is now believed to have originated in C-rich red giant stars during the AGB phase of evolution. This conclusion is supported both by the close similarity of the measured distributions of ${ }^{12} \mathrm{C} /{ }^{13} \mathrm{C}$ ratios (mainly between 20 and 100) in the grains and the stars, and by stunning agreement with models of AGB stars of the isotopic compositions of heavy elements such as $\mathrm{Ba}, \mathrm{Zr}$, and Mo, measured in individual grains by resonance ionization mass spectrometry (e.g., Lugaro et al., 2003). However, the good agreement between the grain compositions and those observed or expected for AGB stars does not extend to the 50\% of the grains' atoms that are $\mathrm{Si}$ ! The Si-isotopic ratios, expressed as $\delta$ values, for the mainstream $\mathrm{SiC}$ grains are shown in Fig. 6. The grains form a linear array of slope 1.3 on this plot, with isotopic ratios ranging from $\sim 0.95$ to $1.2 \times$ solar. In AGB stars, heavy element isotopic compositions can be modified by $\mathrm{n}$-capture reactions in the He-burning shell followed by convective mixing with the stellar envelope. As shown in Fig. 6a, this mixing results in Si-isotopic compositions distinct from the observed grain trend. The slope of the grain data is much steeper than predicted $\mathrm{Si}$-isotopic evolution for single AGB stars [ 0.3-0.8 (Lugaro et al., 1999)]. Moreover, the range of ratios is larger than that predicted for low-mass, solar-metallicity AGB stars $(<4 \%$ shifts in ratios, compared to the observed $25 \%$ range). It is now believed that the mainstream Si array reflects a spread in the initial compositions of a large number of individual stellar sources (Alexander, 1993; Gallino et al., 1994; Timmes and Clayton, 1996; Alexander and Nittler, 1999).

In many presolar $\mathrm{SiC}$ grains, $\mathrm{Ti}$ is in high enough abundance to determine its isotopic composition. Titanium-isotopic measurements of mainstream $\mathrm{SiC}$ grains have indicated a similar behavior to that of Si. Namely, the $46,47,49,50 \mathrm{Ti} / 48 \mathrm{Ti}$ ratios are correlated with the Si-isotopic ratios, forming arrays on three-isotope plots with slopes distinct from those predicted for the mixing of He-shell material with the envelopes of individual AGB stars (Ireland et al., 1991; Hoppe et al., 1994; Alexander and Nittler, 1999; Lugaro et al., 1999). This is illustrated in Fig. 6b, showing the observed correlation between ${ }^{29} \mathrm{Si} / 28 \mathrm{Si}$ and ${ }^{46} \mathrm{Ti} / 48 \mathrm{Ti}$ [see Fig. 4 of Lugaro et al. (1999) for all Ti ratios]. Note that ${ }^{49} \mathrm{Ti}$ and ${ }^{50} \mathrm{Ti}$ are in fact more strongly affected by $\mathrm{n}$-capture in AGB stars than are the $\mathrm{Si}$ and other $\mathrm{Ti}$ isotopes, so that the ${ }^{49} \mathrm{Ti} /$ ${ }^{48} \mathrm{Ti}$ and ${ }^{50} \mathrm{Ti} / 48 \mathrm{Ti}$ ratios are somewhat less strongly correlated with Si-isotopic ratios in the mainstream grains.

The most obvious explanation for a spread in initial isotopic compositions of individual stars is that it reflects some sort of GCE process. As discussed in the previous section, GCE theory predicts that ${ }^{29} \mathrm{Si} / 28 \mathrm{Si}$ and ${ }^{30} \mathrm{Si} / 28 \mathrm{Si}$ ratios increase as the metallicity of the ISM does (Fig. 5). Galactic chemical evolution predictions of Ti isotopes are much less secure than those of Si due to lingering uncertainties in the nucleosynthesis processes responsible for some of them (Timmes et al., 1995). Nonetheless, ${ }^{48} \mathrm{Ti}$ is believed to be a primary isotope while the rarer ${ }^{46} \mathrm{Ti},{ }^{47} \mathrm{Ti},{ }^{49} \mathrm{Ti}$, and ${ }^{50} \mathrm{Ti}$ are 
secondary ones, and the ${ }^{\mathrm{i}} \mathrm{Ti} / 48 \mathrm{Ti}$ ratios probably increase with metallicity.

The renormalized $\mathrm{Si}$ - and Ti-isotope GCE predictions of Timmes et al. (1995) and Timmes and Clayton (1996) are plotted with the SiC data in Fig. 6. Clearly, GCE predicts a better fit to the grain data than does He-shell mixing in single AGB stars. A GCE interpretation of the $\mathrm{Si}$ and Ti data is further supported by the rare $\mathrm{SiC}$ subgroups known as $\mathrm{Y}$ and $\mathrm{Z}$ grains (Meyer and Zinner, 2006). Y grains ( 2\%) have ${ }^{12} \mathrm{C} /{ }^{13} \mathrm{C}$ ratios higher than mainstream grains and $\mathrm{Si}$-isotopic compositions that plot to the right of the mainstream grains in Fig. 6a. These grains are believed to have originated in AGB stars of $\sim 0.5 \mathrm{Z}_{\odot}$ (Amari et al., 2001). When a component due to dredge-up of He-shell material is subtracted from their Si-isotopic compositions, they are inferred to have lower initial ${ }^{29} \mathrm{Si} / 28 \mathrm{Si}$ and ${ }^{30} \mathrm{Si} / 28 \mathrm{Si}$ ratios than mainstream grains, as expected for lower metallicity stars. $\mathrm{Z}$ grains have similar $\mathrm{C}$ isotopes to mainstream grains, but have ${ }^{29} \mathrm{Si}$ depletions and ${ }^{30} \mathrm{Si}$ enrichments, relative to mainstream grains (Hoppe et al., 1997). These grains most likely formed in AGB stars of even lower metallicity than did the Y grains, perhaps as low as $1 / 3 Z_{\odot}$ (Hoppe et al., 1997; Nittler and Alexander, 2003), and their inferred initial Si-isotopic ratios are even lower than the Y grains, consistent with GCE expectations. Moreover, Ti-isotopic measurements of several $\mathrm{Z}$ grains indicate depletions of ${ }^{46} \mathrm{Ti},{ }^{47} \mathrm{Ti}$, and ${ }^{49} \mathrm{Ti}$, relative to solar (Amari et al., 2003; Zinner et al., 2005a), as expected if Ti-isotopic ratios decrease with decreasing metallicity.

There are significant problems with a simple homogeneous GCE interpretation of the $\mathrm{SiC}$ data, however, as illustrated in Fig. 6. First, the slope of the mainstream line is 1.3 , steeper than the slope- 1 line predicted by GCE theory. Second, most of the grains are enriched in the secondary, neutron-rich isotopes relative to the Sun, but formed in stars born earlier than the Sun. In a homogeneous GCE model that produces the Sun, older stars should have ${ }^{29,30} \mathrm{Si} / 28 \mathrm{Si}$ ratios lower than the Sun. Finally, taking the Timmes and Clayton (1996) GCE calculation at face value, the mainstream $\mathrm{SiC}$ data require a difference of about 5 G.y. to explain the range of $\mathrm{Si}$ isotopes from the bottom to the top of the mainstream. From stellar evolutionary considerations, it seems most likely that the $\mathrm{SiC}$ grains originated in stars at least as massive as $\sim 1.5-2 \mathrm{M}_{\odot}$ (Lugaro et al., 1999). Such stars have evolutionary timescales much shorter than 5 G.y., so the time difference indicated by the GCE model would indicate an exceedingly large range in interstellar residence ages of the grains themselves. This hardly seems credible, since there are many ISM processes destructive to dust grains (Jones et al., 1997), making a simple temporal interpretation of the Si data implausible. Even if a temporal interpretation is discarded, the grains still appear to have originated in donor AGB stars with higher initial metallicities than the Sun and the problem of the 1.3 slope remains. A number of attempts have been made to resolve these problems, while maintaining a GCE interpretation of the data.

Clayton and Timmes (1997) postulated that the Sun's Siisotopic composition is strongly peculiar, compared to the mean GCE of the ISM. If so, then the slope-1 line on a Si three-isotope plot predicted by GCE theory could be rotated into a slope-1.3 line as observed for the grains, when normalized to the unusual solar isotope ratios. For this to work quantitatively requires that the Sun must lie far to the right ( ${ }^{30} \mathrm{Si}$-rich) side of the initial GCE line in Fig. 6a. However, in this case dredge-up of He-shell material in the parent AGB stars would have to increase the surface ${ }^{30} \mathrm{Si} / 28 \mathrm{Si}$ ratio so much that the final (observed mainstream) line falls, as if by a miracle, very near the Sun's abnormal composition. Moreover, such a large increase of ${ }^{30} \mathrm{Si} / 28 \mathrm{Si}$ in AGB stars is not regarded as possible, based both on the grains' C-isotopic ratios (Alexander and Nittler, 1999) and on AGB nucleosynthesis calculations (Lugaro et al., 1999).

A second approach, proposed by Clayton (1997), invokes outward diffusion of stars from the metal-rich inner regions of the galaxy. He suggested that the $\mathrm{SiC}$ parent stars formed on circular orbits at smaller galactocentric radii than did the Sun and subsequently scattered from massive molecular clouds into more elongated orbits, eventually ending their lives (during their AGB phases) near the radius of solar birth. Because metallicity gradients exist in the galactic disk, these stars could have higher metallicities than the Sun, despite forming earlier. Stellar orbital diffusion models like this have been advanced to explain the large scatter in metallicity for local disk stars of the same age (Edvardsson et al., 1993; François and Matteucci, 1993; Wielen et al., 1996). A semianalytic model by Nittler and Alexander (1999), using astronomically derived parameters, indicates that such outward orbital diffusion of stars probably would not result in the observed Si-isotopic distribution of the $\mathrm{SiC}$ grains. Moreover, unpublished Monte Carlo calculations (D. D. Clayton, personal communication, 2004) do not indicate large-scale outward scattering of presolar AGB stars.

Alexander and Nittler (1999) took a different approach, attempting to use the grain data themselves to directly infer the relative GCE trends of the isotopic ratios. They took advantage of the fact that the different isotope ratios of $\mathrm{Si}$ and $\mathrm{Ti}$ are affected to differing degrees by $\mathrm{n}$-capture in the AGB He-shell and performed a $\chi^{2}$ fit to the mainstream SiC $\mathrm{Si}$ - and Ti-isotopic data. The composition of each grain was assumed to be a linear mixture of an initial composition and a He-shell composition, predicted by an AGB nucleosynthesis model (Gallino et al., 1994). Contrary to what was claimed in their paper, this fit cannot uniquely determine the relationship between isotopic ratios and metallicity. However, it can infer the relative isotopic ratio GCE trends, i.e., the slopes of mean GCE trends on three-isotope plots. Based on their fit, Alexander and Nittler concluded that the true slope of the Si-isotope GCE trend on the $\mathrm{Si} \delta$-value plot is closer to 1.5 than to 1 . One possibility to obtain such a slope would be that the initial mass function changes with time in the galaxy, since high-mass supernovae models produce $\mathrm{Si}$ with ${ }^{29} \mathrm{Si} /{ }^{30} \mathrm{Si}>$ solar and low-mass ones produce ${ }^{29} \mathrm{Si} /{ }^{30} \mathrm{Si}<$ solar (Woosley and Weaver, 1995). Alternatively, a faster evolution of ${ }^{29} \mathrm{Si}$ than ${ }^{30} \mathrm{Si}$ near solar metallicity would imply a faster evolution of ${ }^{30} \mathrm{Si}$ at low metallicities. 
Thus, if there were a large source of ${ }^{30} \mathrm{Si}$ relative to ${ }^{29} \mathrm{Si}$ at low metallicity, the required steep slope on the Si three-isotope plot might be obtained. There is no hint of an "extra" source of ${ }^{30} \mathrm{Si}$ in low-metallicity supernova calculations, but other possibilities include ONe novae and low-metallicity AGB stars. Recent calculations of each of these stellar types indicate relatively large production factors of ${ }^{30} \mathrm{Si}$ and smaller or no production of ${ }^{29} \mathrm{Si}$ (Amari et al., 2001; José and Hernanz, 1998). Galactic chemical evolution calculations taking these sources into account to test this idea are still lacking.

An alternative approach was anticipated by Timmes and Clayton (1996), who showed that when the nucleosynthetic yields from supernovae are normalized to the solar-metallicity ISM composition calculated by their GCE model, heterogeneous mixing of individual supernova ejecta into an initially $\sim$ solar composition could possibly reproduce a slope 1.3 line on the Si three-isotope plot. Lugaro et al. (1999) followed up on this suggestion and explicitly modeled, using a Monte Carlo technique, the effect of inhomogeneous mixing of SN ejecta into localized regions of the ISM. They showed that with a range of model parameters, the model could easily explain the range and scatter of mainstream $\mathrm{SiC}$ $\mathrm{Si}$-isotope ratios. They further argued that the observed distribution of $\mathrm{SiC} \mathrm{Si} \mathrm{isotopes} \mathrm{probably} \mathrm{reflects} \mathrm{an} \mathrm{interplay}$ of homogeneous GCE gradually increasing the average ISM ${ }^{29,30} \mathrm{Si} / 28 \mathrm{Si}$ ratios and heterogeneous GCE leading to local variations about the mean, although the balance between heterogeneous and homogeneous GCE in shaping the mainstream distribution would depend on the range of ages of the parent stars. An attractive feature of this model is that it can naturally account for the isotopic heaviness of the grains with respect to the Sun. However, Nittler (2005) extended this model to $\mathrm{Ti}$ and $\mathrm{O}$ isotopes and showed that it could explain neither the high degree of correlation between $\mathrm{Si}$ and $\mathrm{Ti}$ isotopes in the grains nor the range of O-isotopic compositions observed in presolar oxide grains (see next section). The observed correlation between ${ }^{29} \mathrm{Si} / 28 \mathrm{Si}$ and ${ }^{46} \mathrm{Ti} / 48 \mathrm{Ti}$ ratios in the grains allows at most $\sim 40 \%$ of the total spread in Si-isotopic composition to be explained by this specific heterogeneous GCE model. It is also highly unlikely that any model of this sort could account for the SiTi correlations because the isotopes of these elements are made in different types and/or masses of supernovae and the isotope ratios in the ejecta of supernovae of different masses and types are hence uncorrelated. In fact, the observed Si-Ti isotope correlations indicate that the disk ISM in the vicinity of solar birth 4.6 G.y. ago was remarkably well-mixed with regard to the ejecta of individual supernovae.

Recently, Clayton (2003) suggested a radically different explanation for the mainstream $\mathrm{SiC} \mathrm{Si-} \mathrm{(and} \mathrm{Ti-)} \mathrm{isotopic}$ ratio distribution. In his model, the isotopic correlation lines are two-component mixing lines due to a merger of a lowmetallicity dwarf galaxy with the Milky Way disk some 67 G.y. ago. At that time, Clayton postulates that the ${ }^{29} \mathrm{Si} /$ ${ }^{28} \mathrm{Si}$ and ${ }^{30} \mathrm{Si} / 28 \mathrm{Si}$ ratios of the local Milky Way ISM were higher than the solar ratios and the merging galaxy had lower-than-solar ratios. The merger induced a period of star formation during which the $\mathrm{SiC}$ parent stars were born with a range of initial isotopic compositions due to variable mixing of the interstellar gas from the two components. Since the Sun formed in the same region of the disk, it incorporated its own mix of the two galaxies as well as the local ejecta of stars that occurred between the time of merger and that of solar birth. This model has many attractions, including explanations for the tight correlation between the mainstream $\mathrm{SiC} \mathrm{Si-}$ and $\mathrm{Ti}$-isotope ratios, the placement of the $\mathrm{Sun}$ at the bottom of the $\mathrm{SiC}$ mainstream, and the unusual ${ }^{18} \mathrm{O} /{ }^{17} \mathrm{O}$ ratio of the Sun, compared with the local ISM (Penzias, 1981). Moreover, mergers like that postulated are now commonly believed to be how much of the mass of the galaxy was acquired (e.g., Shetrone et al., 2003; Wyse, 2003). However, it clearly needs much critical scrutiny by assorted scientific disciplines before it can be accepted. For example, it might be possible to find a fossil record of such a merger in the chemical compositions of nearby stars formed at that time.

We note that the rare $\mathrm{SiC} \mathrm{Y}$ grains might provide some support for the galactic merger model outlined above (Clayton, 2003). The initial Si-isotopic compositions of most Y grains are inferred to lie near the lower end of the mainstream line. Moreover, there is a smooth and rapid increase in the fraction of $\mathrm{SiC}$ grains with high ${ }^{12} \mathrm{C} /{ }^{13} \mathrm{C}$ (a rough proxy for AGB mass) with decreasing ${ }^{29} \mathrm{Si} / 28 \mathrm{Si}$ ratios (Nittler and Alexander, 2003). Because more-massive stars evolve faster than less-massive ones, these results imply a more recent formation for the parent stars of grains near the bottom of the mainstream compared with those with higher ${ }^{29,30} \mathrm{Si} / 28 \mathrm{Si}$ ratios. This runs counter to the expectations for the temporal galactic evolution interpretation of the mainstream line, but is compatible with the physical mixing model, assuming that the mixing fraction of the accreting dwarf galaxy material increases with time during the merger event.

\subsection{Galactic Chemical Evolution and Presolar Oxide Grains}

Galactic chemical evolution has also been implicated in interpretations of isotope data for presolar oxide grains in meteorites, primarily corundum, $\mathrm{Al}_{2} \mathrm{O}_{3}$, and spinel, $\mathrm{MgAl}_{2} \mathrm{O}_{4}$ (e.g., Nittler et al., 1997; Choi et al., 1998; Meyer and Zinner, 2006). The O-isotopic ratios for several hundred identified presolar oxide grains are plotted in Fig. 7; most of the grains have been assigned to four groups by Nittler et al. (1997). We focus here on the Group 1 and 3 grains, since, as discussed below, these provide information about GCE. The initial O-isotopic compositions of the parent stars of the highly ${ }^{18} \mathrm{O}$-depleted Group 2 grains are believed to have been completely erased by nucleosynthetic processes and the origin of the ${ }^{18} \mathrm{O}$-rich Group 4 grains is enigmatic (Meyer and Zinner, 2006).

The majority of the presolar oxide grains are believed to have formed in low-mass red giant and AGB stars. In par- 


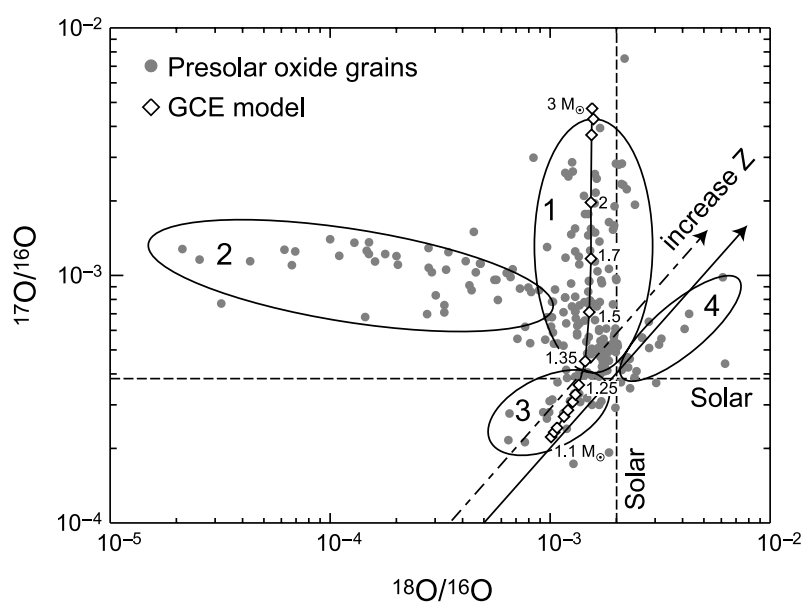

Fig. 7. Oxygen-isotopic ratios measured in presolar oxide minerals (mostly $\mathrm{Al}_{2} \mathrm{O}_{3}$ and $\mathrm{MgAl}_{2} \mathrm{O}_{4}$ ) from meteorites. Ellipses indicate group definitions of Nittler et al. (1997). Arrows indicate the expected GCE of $\mathrm{O}$ isotopes in the ISM (solid line: ${ }^{18} \mathrm{O} /{ }^{17} \mathrm{O}=$ 5.2; dash-dot arrow: ${ }^{18} \mathrm{O} /{ }^{17} \mathrm{O}=3.5$ ). Open diamonds indicate predicted average O-isotopic compositions of presolar red giant stars of different masses, taking into account GCE, the first dredge-up, and stellar lifetimes (Nittler et al., 1997); stellar masses are indicated for some points. The good agreement between the GCE model and the Group 1 and 3 oxide grains indicates that these grains formed in red giant stars and that the ${ }^{18} \mathrm{O} /{ }^{17} \mathrm{O}$ ratio of the Sun is not atypical for its age and location in the galaxy. Data from Nittler (1997, and references therein), Choi et al. (1998), and Zinner et al. (2003).

ticular, the O-isotopic ratios of the dominant Group 1 grains are consistent both with spectroscopic observations of $\mathrm{O}-$ rich red giants and AGB stars (Harris and Lambert, 1984) and with model calculations of nucleosynthesis and mixing processes in these stars (Boothroyd and Sackmann, 1999; Dearborn, 1992; El Eid, 1994). The ${ }^{17} \mathrm{O} /{ }^{16} \mathrm{O}$ ratios of these grains are explained by a range of masses of the parent stars, but the range of ${ }^{18} \mathrm{O} /{ }^{16} \mathrm{O}$ ratios is larger than can be explained by mixing processes in the stars themselves. Analogous to the $\mathrm{Si}$ isotopes in $\mathrm{SiC}$ grains, a range of initial ${ }^{18} \mathrm{O}$ / ${ }^{16} \mathrm{O}$ ratios in the parent stars is required. As discussed in section 5, this ratio is expected to increase with time in the galaxy, so GCE is an obvious explanation for the data.

Models of O-isotopic GCE have been reported over the last decade (Timmes et al., 1995; Prantzos et al., 1996; Romano and Matteucci, 2003). Unfortunately, although the more recent models can well explain the ${ }^{17} \mathrm{O} /{ }^{16} \mathrm{O}$ ratio of both the Sun and of molecular clouds throughout the galaxy, a quantitative understanding of the GCE of the ${ }^{18} \mathrm{O} / 16 \mathrm{O}$ ratio is still lacking. For example, there are gradients with galactocentric radius in the molecular cloud ${ }^{17} \mathrm{O} /{ }^{16} \mathrm{O}$ and ${ }^{18} \mathrm{O} /{ }^{16} \mathrm{O}$ ratios, but no such gradient in the ${ }^{18} \mathrm{O} / 17 \mathrm{O}$ ratio (Penzias, 1981; Wilson and Rood, 1994). This is consistent with the expected primary(secondary) behavior of ${ }^{16} \mathrm{O}\left({ }^{17,18} \mathrm{O}\right)$, but the molecular cloud ${ }^{18} \mathrm{O} /{ }^{17} \mathrm{O}$ ratio is $\sim 3.5$, much lower than the solar value of 5.2. There is still no good explanation for this discrepancy, although both systematic errors in the molecular cloud observations and a local enrichment by massive supernova ejecta of the solar system's progenitor molecular cloud have been suggested (e.g., Prantzos et al., 1996).

The secondary nature of ${ }^{18} \mathrm{O}$ implies that presolar oxide grains with lower ${ }^{18} \mathrm{O} / 16 \mathrm{O}$ ratios originated in stars with lower metallicity than the parent stars of grains with higher ratios. However, the precise relationship between ${ }^{18} \mathrm{O} / 16 \mathrm{O}$ and metallicity is unknown. Nittler et al. (1997) presented a simple model to explain the distribution of O-isotopic ratios of Group 1 and 3 presolar oxide grains. This model predicted the average ${ }^{17} \mathrm{O} / 16 \mathrm{O}$ and ${ }^{18} \mathrm{O} / 16 \mathrm{O}$ ratios of red giant stars as a function of mass (from $\sim 1.2$ to $3 \mathrm{M}_{\odot}$ ), such that the stars ended their life 4.6 G.y. ago. Lower-mass stars formed earlier in galactic history (since they have longer lifetimes) than higher-mass stars and hence would be expected to have lower metallicity and lower initial ${ }^{18} \mathrm{O} /{ }^{16} \mathrm{O}$ ratios. Highermass stars also are predicted to have higher ${ }^{17} \mathrm{O} /{ }^{16} \mathrm{O}$ ratios following mixing of interior material to the stellar surface during the red giant phase of evolution ("first dredge-up"). For lack of a good O-isotopic GCE trend, this model assumed that the ${ }^{17} \mathrm{O} / 16 \mathrm{O}$ and ${ }^{18} \mathrm{O} / 16 \mathrm{O}$ ratios in the ISM increase linearly with metallicity (Boothroyd and Sackmann, 1999 ) and that at solar metallicity the solar ${ }^{18} \mathrm{O} /{ }^{17} \mathrm{O}$ (rather than the molecular cloud one) is reproduced. Predictions of the resulting model are shown on Fig. 7; the model curve passes through the center of the distribution of both Group 1 and 3 oxide grains. This result strongly suggests that the Group 3 grains also formed in red giants and AGB stars, albeit ones with initial O-isotopic ratios lower than solar. Moreover, note that the first dredge-up in red giants can only decrease the surface ${ }^{18} \mathrm{O} /{ }^{17} \mathrm{O}$ ratio. Thus, if the typical presolar ISM had an ${ }^{18} \mathrm{O} /{ }^{17} \mathrm{O}$ ratio similar to that observed today in molecular clouds, one would expect all Group 1 and 3 presolar oxide grains to have ${ }^{18} \mathrm{O} /{ }^{17} \mathrm{O}<3.5$. But in fact, some $30 \%$ of the grains have ratios higher than this value. Thus the grain data imply that typical ISM ${ }^{18} \mathrm{O} /{ }^{17} \mathrm{O}$ ratios were greater in presolar times than is observed today in molecular clouds throughout the galactic disk, perhaps indicating a problem with the molecular cloud observations themselves. Alternatively, the galactic merger model proposed by Clayton (2003) to explain mainstream $\mathrm{SiC}$ grains (previous section) might also explain the discrepancy between ${ }^{18} \mathrm{O} /{ }^{17} \mathrm{O}$ in the present-day ISM and in the Sun.

Because low-mass stars have long evolutionary timescales and the parent stars of the presolar grains must have ended their lives prior to the formation of the Sun 4.6 G.y. ago, the grain data also can be used to constrain the age of our galaxy (Nittler and Cowsik, 1997). The age of the galaxy must be larger than the longest lifetime of a grain parent star added to that of the Sun. Nittler and Cowsik (1997) calculated a lower bound on the age of the disk as 10.5 G.y. and an actual age of 14.4 G.y. The systematic uncertainties affecting this estimate are potentially large (several gigayears), but the age agrees well with those estimated by other means and was determined in a fundamentally new way. 
Since the distribution of ${ }^{18} \mathrm{O} / 16 \mathrm{O}$ ratios observed in the Group 1 and 3 presolar oxide grains is in good agreement with expectations for GCE, the metallicities of the parent stars can be estimated from grain O-isotope ratios and theoretical models. The grains can then be used to trace evolutionary histories of other isotope systems, if the relevant isotope measurements can be made on the grains. For example, $\mathrm{Mg}$ is another element that has both primary $\left({ }^{24} \mathrm{Mg}\right)$ and secondary $\left({ }^{25} \mathrm{Mg},{ }^{26} \mathrm{Mg}\right)$ isotopes. Both theoretical models (e.g., Timmes et al., 1995) and spectroscopic observations of main-sequence stars (e.g., Gay and Lambert, 2000)

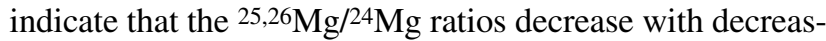
ing metallicity, but the stellar data have large error bars. Presolar grains often have large ${ }^{26} \mathrm{Mg}$ excesses due to in situ decay of radioactive ${ }^{26} \mathrm{Al}$ (Meyer and Zinner, 2006). Measurements of ${ }^{25} \mathrm{Mg} / 24 \mathrm{Mg}$ ratios in mainstream $\mathrm{SiC}$ grains (Huss et al., 1997) revealed no variations due to GCE or otherwise, but because $\mathrm{Mg}$ contents are very low in the grains, terrestrial contamination cannot be ruled out. Recently, Mg-isotopic data have been reported for a number of presolar $\mathrm{MgAl}_{2} \mathrm{O}_{4}$ grains (Nittler et al., 2003; Zinner et al., 2005b). Although the isotopic systematics of the grains are complex, the ${ }^{25} \mathrm{Mg} / 24 \mathrm{Mg}$ ratios suggest that the evolution of this ratio as a function of metallicity is relatively shallow near solar metallicity. This is consistent both with recent observational data (Gay and Lambert, 2000) and GCE models taking AGB star nucleosynthesis into account (Fenner et al., 2003).

Titanium-isotopic ratios have been measured in a few presolar $\mathrm{Al}_{2} \mathrm{O}_{3}$ grains as well (Choi et al., 1998; Hoppe et al., 2003). Because of low Ti concentrations, error bars for most reported measurements are large. However, a few grains have large anomalies and/or small error bars and allow a comparison with $\mathrm{SiC} \mathrm{Ti}$ data. In general, the observations are consistent with the GCE interpretation of $\mathrm{Ti}$ isotopes in $\mathrm{SiC}$ (section 6.1) (Alexander and Nittler, 1999). For example, there is a general correlation between ${ }^{46} \mathrm{Ti} / 48 \mathrm{Ti}$ and ${ }^{18} \mathrm{O} / 16 \mathrm{O}$ as expected since both are secondary/primary isotope ratios.

\section{CONCLUDING REMARKS}

It might seem audacious to attempt to use microscopic constituents of rare and unusual rocks to draw broad inferences about the vast reaches of space in time encompassed by the chemical history of the Milky Way. However, it is clear at this point that galactic evolution has left an isotopic record within meteorites, both in the fossil remnants of radioactive nuclei and in presolar grains of stardust. Our attempts to decode this record are, of course, far from complete. For example, although a signature of GCE is clearly imprinted on some isotopic ratios measured in presolar grains, the exact process or processes involved (e.g., homogeneous GCE, heterogeneous GCE, a galactic merger) have not been unambiguously identified. Full exploitation of the potential of the grains for GCE science will require both additional theoretical and observational work. For example, a presolar galactic merger in the solar neighborhood should have left some record in the chemical compositions of the stars formed at the time. Moreover, GCE models including the relevant isotopic ratios and current nucleosynthetic yields from all types of stars (including, for instance, novae and low-Z AGB stars) are sparse or lacking. There will no doubt be considerable progress in the coming decades as the sophistication of computer models increases as well as new astronomical and meteoritical data are obtained by advances in technology.

As mentioned in section 1, the solar chemical composition was long considered the "cosmic" composition, and a key question in cosmochemistry remains: Just how typical is the solar composition in the context of the galactic evolution? Based on spectroscopic determination of many elements in the atmospheres of dwarf stars in the solar neighborhood, Edvardsson et al. (1993) concluded that the Sun's composition is quite typical for its age and location in the galaxy. Moreover, the metallicity distribution for $\mathrm{G}$ dwarfs in the solar neighborhood (Nordström et al., 2004) has a peak very close to the solar value $([\mathrm{Me} / \mathrm{H}]=0 ;$ Fig. 2$)$. A very important observation to be gleaned from section 6 is that the solar composition is also apparently isotopically quite typical. Most presolar SiC grains formed in stars with initial Si-isotopic compositions within $\sim 25 \%$ of the solar value and the distribution of $\mathrm{O}$ isotopes in presolar oxide grains indicate that their parent stars had ${ }^{18} \mathrm{O} /{ }^{17} \mathrm{O}$ ratios closer to the solar value than to that measured today in molecular clouds. Furthermore, trace heavy elements in individual presolar grains from AGB stars (Nicolussi et al., 1997; Lugaro et al., 2003) have isotopic compositions consistent with mixing of He-shell material with envelope material of essentially solar isotopic composition. Thus, if nothing else, the meteorite data discussed in this chapter help confirm the utility of using the solar composition, about which we know so much, as a standard with which to compare the chemical makeup of the rest of the remote universe, about which we know much less.

Acknowledgments. This work benefited from many fruitful discussions with C. Alexander, D. D. Clayton, A. M. Davis, R. Gallino, T. Rauscher, B. Marty, L. Reisberg, F. Timmes, J. W. Truran, M. Wadhwa, and R. Yokochi. This chapter was improved by constructive reviews by B. Meyer and E. Zinner.

\section{REFERENCES}

Alexander C. M. O’D. (1993) Presolar SiC in chondrites: How variable and how many sources? Geochim. Cosmochim. Acta, 57, 2869-2888.

Alexander C. M. O'D. and Nittler L. R. (1999) The galactic evolution of $\mathrm{Si}$, Ti, and $\mathrm{O}$ isotopic ratios. Astrophys. J., 519, 222235.

Allende Prieto C., Lambert D. L., and Asplund M. (2001) The forbidden abundance of oxygen in the sun. Astrophys. J. Lett., 556, L63-L66.

Amari S., Nittler L. R., Zinner E., Gallino R., Lugaro M., and Lewis R. S. (2001) Presolar SiC grains of type Y: Origin from 
low-metallicity asymptotic giant branch stars. Astrophys. J., 546, 248-266.

Amari S., Zinner E., Gallino R., and Lewis R. S. (2003) Silicon and titanium isotopic analysis of silicon carbide grains of type $\mathrm{X}$ and Z (abstract). Meteoritics \& Planet. Sci., 38, A66.

Amelin Y., Krot A. N., Hutcheon I. D., and Ulyanov A. A. (2002) Lead isotopic ages of chondrules and calcium-aluminum-rich inclusions. Science, 297, 1678-1683.

Anders E. and Grevesse N. (1989) Abundances of the elements: Meteoritic and solar. Geochim. Cosmochim. Acta, 53, 197-214.

Argast D., Samland M., Gerhard O. E., and Thielemann F.-K. (2000) Metal-poor halo stars as tracers of ISM mixing processes during halo formation. Astron. Astrophys., 356, 873-887.

Arlandini C., Käppeler F., Wisshak K., Gallino R., Lugaro M., Busso M., and Straniero O. (1999) Neutron capture in lowmass asymptotic giant branch stars: Cross sections and abundance signatures. Astrophys. J., 525, 886-900.

Bernatowicz T., Fraundorf G., Tang M., Anders E., Wopenka B., Zinner E., and Fraundorf P. (1987) Evidence for interstellar SiC in the Murray carbonaceous meteorite. Nature, 330, 728-730.

Birck J. L. and Allègre C. J. (1985) Evidence for the presence of ${ }^{53} \mathrm{Mn}$ in the early solar system. Geophys. Res. Lett., 12, 745748.

Boothroyd A. I. and Sackmann I.-J. (1999) The CNO-isotopes: Deep circulation in red giants and first and second dredge-up. Astrophys. J., 510, 232.

Brazzle R. H., Pravdivtseva O., Meshik A., and Hohenberg C. (1999) Verification and interpretation of the I-Xe chronometer. Geochim. Cosmochim. Acta, 63, 739-760.

Brook C. B., Kawata D., and Gibson B. K. (2003) Simulating a white dwarf dominated galactic halo. Mon. Not. R. Astron. Soc., 343, 913-923.

Burbidge E. M., Burbidge G. R., Fowler W. A., and Hoyle F. (1957) Synthesis of the elements in stars. Rev. Mod. Phys., 29, 547-650.

Cameron A. G. W. and Truran J. W. (1977) The supernova trigger for formation of the solar system. Icarus, 30, 447-461.

Cameron A. G. W., Höflich P., Myers P. C., and Clayton D. D. (1995) Massive supernovae, Orion gamma rays, and the formation of the solar system. Astrophys. J. Lett., 447, L53-L57.

Cayrel R., Hill V., Beers T. C., Barbuy B., Spite M., Spite F., Plez B., Andersen J., Bonifacio P., François P., Molaro P., Nordström B., and Primas F. (2001) Measurement of stellar age from uranium decay. Nature, 409, 691-692.

Chang R. X., Hou J. L., Shu C. G., and Fu C. Q. (1999) Twocomponent model for the chemical evolution of the galactic disk. Astron. Astrophys., 350, 38-48.

Chen J. H. and Wasserburg G. J. (1981) The isotopic composition of uranium and lead in Allende inclusions and meteorite phosphates. Earth Planet. Sci. Lett., 52, 1-15.

Chen J. H. and Wasserburg G. J. (1996) Live ${ }^{207} \mathrm{Pd}$ in the early solar system and implications for planetary evolution. In Earth Processes: Breaking the Isotopic Code (A. Basu and S. Hart, eds.), pp. 1-20. AGU Geophysical Monograph 95, American Geophysical Union, Washington, DC.

Chen J. H., Wasserburg G. J., and Papanastassiou D. A. (1993) Th and $U$ abundances in chondritic meteorites (abstract). In Lunar and Planetary Science XXIV, pp. 277-278. Lunar and Planetary Institute, Houston.

Chiappini C., Matteucci F., and Gratton R. (1997) The chemical evolution of the galaxy: The two-infall model. Astrophys. J., 477, 765-780.
Choi B.-G., Huss G. R., Wasserburg G. J., and Gallino R. (1998) Presolar corundum and spinel in ordinary chondrites: Origins from AGB stars and a supernova. Science, 282, 1284-1289.

Clayton D. D. (1983) Extinct radioactivities - a three-phase mixing model. Astrophys. J., 268, 381-384.

Clayton D. D. (1985) Galactic chemical evolution and nucleocosmochronology: A standard model. In Nucleosynthesis: Challenges and New Developments (W. D. Arnett and J. W. Truran, eds.), pp. 65-88. Univ. of Chicago, Chicago.

Clayton D. D. (1988a) Nuclear cosmochronology within analytic models of the chemical evolution of the solar neighbourhood. Mon. Not. R. Astron. Soc., 234, 1-36.

Clayton D. D. (1988b) Isotopic anomalies: Chemical memory of galactic evolution. Astrophys. J., 334, 191-195.

Clayton D. D. (1997) Placing the sun and mainstream SiC particles in galactic chemodynamical evolution. Astrophys. J. Lett., 484, L67-L70.

Clayton D. D. (2003) A presolar galactic merger spawned the SiCgrain mainstream. Astrophys. J., 598, 313-324.

Clayton D. D. and Nittler L. R. (2004) Astrophysics with presolar stardust. Annu. Rev. Astron. Astrophys., 42, 39-78.

Clayton D. D. and Timmes F. X. (1997) Placing the Sun in galactic chemical evolution: Mainstream $\mathrm{SiC}$ particles. Astrophys. J., 483, 220-227.

Clayton R. N. (1993) Oxygen isotopes in meteorites. Annu. Rev. Earth Planet. Sci., 21, 115-149.

Clayton R. N. (2002) Solar system: Self-shielding in the solar nebula. Nature, 415, 860-861.

Clayton R. N., Grossman L., and Mayeda T. K. (1973) A component of primitive nuclear composition in carbonaceous meteorites. Science, 182, 485-488.

Copi C. J. (1997) A stochastic approach to chemical evolution. Astrophys. J., 487, 704-718.

Cowan J. J., Thielemann F.-K., and Truran J. W. (1991) Radioactive dating of the elements. Annu. Rev. Astron. Astrophys., 29, 447-497.

Cowan J. J., Sneden C., Burles S., Ivans I. I., Beers T. C., Truran J. W., Lawler J. E., Primas F., Fuller G. M., Pfeiffer B., and Kratz K.-L. (2002) The chemical composition and age of the metal-poor halo star BD $+17^{\circ} 3248$. Astrophys. J., 572, 861879.

Dauphas N. (2005a) The U/Th production ratio and the radiometric age of the Milky Way. Nature, 439, 1203-1205.

Dauphas N. (2005b) Multiple sources or late injection of shortlived r-nuclides in the early solar system? Nucl. Phys. A, 758, 757-760.

Dauphas N., Rauscher T., Marty B., and Reisberg L. (2003) Shortlived p-nuclides in the early solar system and implications on the nucleosynthetic role of X-ray binaries. Nucl. Phys. A., 719, $287 \mathrm{c}-295 \mathrm{c}$.

Dauphas N., Foley C. N., Wadhwa M., Davis A. M., Janney P. E., Qin L., Göpel C., Birck J.-L. (2005) Protracted core differentiation in asteroids from ${ }^{182} \mathrm{Hf}-182 \mathrm{~W}$ systematics in the Eagle Station pallasite (abstract). In Lunar and Planetary Science XXXVI, Abstract \#1100. Lunar and Planetary Institute, Houston (CDROM).

Dearborn D. S. P. (1992) Diagnostics of stellar evolution: The oxygen isotopes. Phys. Rept., 210, 367-382.

Dopita M. A. and Ryder S. D. (1994) On the law of star formation in disk galaxies. Astrophys. J., 430, 163-178.

Edvardsson B., Anderson J., Gustaffson B., Lambert D. L., Nissen P. E., and Tomkin J. (1993) The chemical evolution of the ga- 
lactic disk. Astron. Astrophys., 275, 101-152.

El Eid M. (1994) CNO isotopes in red giants: Theory versus observations. Astron. Astrophys., 285, 915-928.

Fenner Y., Gibson B. K., Lee H.-c., Karakas A. I., Lattanzio J. C., Chieffi A., Limongi M., and Yong D. (2003) The chemical evolution of magnesium isotopic abundances in the solar neighbourhood. Publ. Astron. Soc. Australia, 20, 340-344.

François P. and Matteucci F. (1993) On the abundance spread in solar neighbourhood stars. Astron. Astrophys., 280, 136-140.

Friedrich J. M., Ott U., and Lugmair G. W. (2004) Revisiting extraterrestrial $\mathrm{U}$ isotope ratios (abstract). In Lunar and Planetary Science $X X X V$, Abstract \#1575. Lunar and Planetary Institute, Houston (CD-ROM).

Gallino R., Raiteri C. M., Busso M., and Matteucci F. (1994) The puzzle of silicon, titanium and magnesium anomalies in meteoritic silicon carbide grains. Astrophys. J., 430, 858-869.

Gallino R., Busso M., Wasserburg G. J., and Straniero O. (2004) Early solar system radioactivity and AGB stars. New Astron. Rev., 48, 133-138.

Gay P. L. and Lambert D. L. (2000) The isotopic abundances of magnesium in stars. Astrophys. J., 533, 260-270.

Gerritsen J. P. E. and Icke V. (1997) Star formation in N-body simulations. I. The impact of the stellar ultraviolet radiation on star formation. Astron. Astrophys., 325, 972-986.

Gibson B. K., Fenner Y., Renda A., Kawata D., and Lee H. (2003) Galactic chemical evolution. Publ. Astron. Soc. Australia, 20, 401-415.

Goreva J. S. and Burnett D. S. (2001) Phosphate control on the $\mathrm{Th} / \mathrm{U}$ variations in ordinary chondrites: Improving solar system abundances. Meteoritics \& Planet. Sci., 36, 63-74.

Goriely S. and Arnould M. (2001) Actinides: How well do we know their stellar production? Astron. Astrophys., 379, 1113 1122.

Grenon M. (1987) Past and present metal abundance gradient in the galactic disc. J. Astrophys. Astron., 8, 123-139.

Hansen B. M. S., Richer H. B., Fahlman G. G., Stetson P. B. Brewer J., Currie T., Gibson B. K., Ibata R., Rich R. M., and Shara M. M. (2004) Hubble Space Telescope observations of the white dwarf cooling sequence of M4. Astrophys. J. Suppl. Ser., 155, 551-576.

Harper C. L. (1996) Evidence for ${ }^{92 \mathrm{~g} N b}$ in the early solar system and evaluation of a new p-process cosmochronometer from ${ }^{92 \mathrm{~g}} \mathrm{Nb} /{ }^{92} \mathrm{Mo}$. Astrophys. J., 466, 437-456.

Harris M. J. and Lambert D. L. (1984) Oxygen isotopic abundances in the atmospheres of seven red giant stars. Astrophys. J., 285, 674-682.

Hill V., Plez B., Cayrel R., Beers T. C., Nordström B., Andersen J., Spite M., Spite F., Barbuy B., Bonifacio P., Depagne E., François P., and Primas F. (2002) First stars. I. The extreme r-element rich, iron-poor halo giant CS 31082-001. Implications for the r-process site(s) and radioactive cosmochronology. Astron. Astrophys., 387, 560-579.

Hirata T. (2001) Determinations of $\mathrm{Zr}$ isotopic composition and $\mathrm{U}-\mathrm{Pb}$ ages for terrestrial $\mathrm{Zr}$-bearing minerals using laser ablation-inductively coupled plasma mass spectrometry: Implications for $\mathrm{Nb}-\mathrm{Zr}$ isotopic systematics. Chem. Geol., 176, 323342.

Hoppe P., Amari S., Zinner E., Ireland T., and Lewis R. S. (1994) Carbon, nitrogen, magnesium, silicon and titanium isotopic compositions of single interstellar silicon carbide grains from the Murchison carbonaceous chondrite. Astrophys. J., 430, 870-890.
Hoppe P., Annen P., Strebel R., Eberhardt P., Gallino R., Lugaro M., Amari S., and Lewis R. S. (1997) Meteoritic silicon carbide with unusual Si-isotopic compositions: Evidence for an origin in low-mass low-metallicity AGB stars. Astrophys. J. Lett., 487, L101-L104.

Hoppe P., Nittler L. R., Mostefaoui S., Alexander C. M. O’D., and Marhas K. K. (2003) A NanoSIMS study of titanium-isotopic compositions of presolar corundum grains (abstract). In Lunar and Planetary Science XXXIV, Abstract \#1570. Lunar and Planetary Institute, Houston (CD-ROM).

Hudson G. B., Kennedy B. M., Podosek F. A., and Hohenberg C. M. (1989) The early solar system abundance of ${ }^{244} \mathrm{Pu}$ as inferred from the St. Severin chondrite. Proc. Lunar Planet. Sci. Conf. 19th, pp. 547-557.

Huss G. R., Hutcheon I. D., and Wasserburg G. J. (1997) Isotopic systematics of presolar silicon carbide from the Orgueil (CI) chondrite: Implications for solar-system formation and stellar nucleosynthesis. Geochim. Cosmochim. Acta, 61, 5117-5148.

Ireland T. R., Zinner E. K., and Amari S. (1991) Isotopically anomalous $\mathrm{Ti}$ in presolar $\mathrm{SiC}$ from the Murchison meteorite. Astrophys. J. Lett., 376, L53-L56.

Jones A. P., Tielens A. G. G. M., Hollenbach D. J., and McKee C. F. (1997) The propagation and survival of interstellar grains. In Astrophysical Implications of the Laboratory Study of Presolar Materials (T. J. Bernatowicz and E. Zinner, eds.), pp. 595613. AIP Conference Proceedings 402, American Institute of Physics, New York.

Jørgensen B. R. (2000) The G dwarf problem. Analysis of a new data set. Astron. Astrophys., 363, 947-957.

José J. and Hernanz M. (1998) Nucleosynthesis in classical novae: CO versus ONe white dwarfs. Astrophys. J., 494, 680-690.

Kelley W. R. and Wasserburg G. J. (1978) Evidence for the existence of Pd-107 in the early solar system. Geophys. Res. Lett., 5, 1079-1082.

Kennicutt R. C. (1998) The global Schmidt law in star-forming galaxies. Astrophys. J., 498, 541-552.

Kotoneva E., Flynn C., Chiappini C., and Matteucci F. (2002) K dwarfs and the chemical evolution of the solar cylinder. Mon. Not. R. Astron. Soc., 336, 879-891.

Krauss L. M. and Chaboyer B. (2003) Age estimates of globular clusters in the Milky Way: Constraints on cosmology. Science, 299, 65-69.

Kravtsov A. V. (2003) On the origin of the global Schmidt law of star formation. Astrophys. J. Lett., 590, L1-L4.

Kroupa P. (2002) The initial mass function of stars: Evidence for uniformity in variable systems. Science, 295, 82-91.

Larson R. B. (1974) Dynamical models for the formation and evolution of spherical galaxies. Mon. Not. R. Astron. Soc., 166, 585-616.

Lewis R. S., Tang M., Wacker J. F., Anders E., and Steel E. (1987) Interstellar diamonds in meteorites. Nature, 326, 160-162.

Lugaro M., Zinner E., Gallino R., and Amari S. (1999) Si isotopic ratios in mainstream presolar $\mathrm{SiC}$ grains revisited. Astrophys. J., 527, 369-394.

Lugaro M., Herwig F., Lattanzio J. C., Gallino R., and Straniero O. (2003) s-process nucleosynthesis in asymptotic giant branch stars: A test for stellar evolution. Astrophys. J., 586, 13051319.

Lugmair G. W. and Shukolyukov A. (1998) Early solar system timescales according to ${ }^{53} \mathrm{Mn}-{ }^{53} \mathrm{Cr}$ systematics. Geochim. Cosmochim. Acta, 62, 2863-2886.

Lugmair G. W., Shimamura T., Lewis R. S., and Anders E. (1983) 
Samarium-146 in the early solar system: Evidence from neodymium in the Allende meteorite. Science, 222, 1015-1018.

Lynden-Bell D. (1975) The chemical evolution of galaxies. Vistas Astron., 19, 299-316.

Matteucci F. (2003) The Chemical Evolution of the Galaxy. Kluwer, Dordrecht. 293 pp.

Matteucci F. and Greggio L. (1986) Relative roles of type I and II supernovae in the chemical enrichment of the interstellar gas. Astron. Astrophys., 154, 279-287.

McWilliam A. (1997) Abundance ratios and galactic chemical evolution. Annu. Rev. Astron. Astrophys., 35, 503-556.

Meyer B. S. and Clayton D. D. (2000) Short-lived radioactivities and the birth of the Sun. Space Sci. Rev., 92, 133-152.

Meyer B. S. and Luo N. (1997) Monte-Carlo modeling of extinct short-lived r-process radioactivities (abstract). In Lunar and Planetary Science XXXIV, Abstract \#1841. Lunar and Planetary Institute, Houston (CD-ROM).

Meyer B. S. and Truran J. W. (2000) Nucleocosmochronology. Phys. Rept., 333-334, 1-11.

Meyer B. S. and Zinner E. (2006) Nucleosynthesis. In Meteorites and the Early Solar System II (D. S. Lauretta and H. Y. McSween Jr., eds.), this volume. Univ. of Arizona, Tucson.

Meyer B. S., The L.-S., Clayton D. D., and El Eid M. F. (2004) Helium-shell nucleosynthesis and extinct radioactivities (abstract). In Lunar and Planetary Science XXXV, Abstract \#1908. Lunar and Planetary Institute, Houston (CD-ROM).

Münker C., Weyer S., Mezger K., Rehkämper M., Wombacher F., and Bischoff A. (2000) ${ }^{92} \mathrm{Nb}-{ }^{92} \mathrm{Zr}$ and the early differentiation history of planetary bodies. Science, 289, 1538-1542.

Nicolussi G. K., Davis A. M., Pellin M. J., Lewis R. S., Clayton R. N., and Amari S. (1997) s-process zirconium in presolar silicon carbide grains. Science, 277, 1281-1283.

Nittler L. R. (1997) Presolar oxide grains in meteorites. In Astrophysical Implications of the Laboratory Studies of Presolar Materials (T. J. Bernatowicz and E. Zinner, eds.), pp. 59-82. AIP Conference Proceedings 402, American Institute of Physics, New York.

Nittler L. R. (2003) Presolar stardust in meteorites: Recent advances and scientific frontiers. Earth Planet. Sci. Lett., 209, 259-273.

Nittler L. R. (2005) Constraints on heterogeneous galactic chemical evolution from meteoritic stardust. Astrophys. J., 618, 281296.

Nittler L. R. and Alexander C. M. O’D. (1999) Can stellar dynamics explain the metallicity distributions of presolar grains? Astrophys. J., 526, 249-256.

Nittler L. R. and Alexander C. M. O'D. (2003) Automated isotopic measurements of micron-sized dust: Application to meteoritic presolar silicon carbide. Geochim. Cosmochim. Acta, 67, 4961-4980.

Nittler L. R. and Cowsik R. (1997) Galactic age estimates from O-rich stardust in meteorites. Phys. Rev. Lett., 78, 175-178.

Nittler L. R., Alexander C. M. O'D., Gao X., Walker R. M., and Zinner E. (1997) Stellar sapphires: The properties and origins of presolar $\mathrm{Al}_{2} \mathrm{O}_{3}$ in meteorites. Astrophys. J., 483, 475-495.

Nittler L. R., Hoppe P., Alexander C. M. O’D., Busso M., Gallino R., Marhas K. K., and Nollett K. (2003) Magnesium isotopes in presolar spinel (abstract). In Lunar and Planetary Science XXXIV, Abstract \#1703. Lunar and Planetary Institute, Houston (CD-ROM).

Nordström B., Mayor M., Andersen J., Holmberg J., Pont F., Jørgensen B. R., Olsen E. H., Udry S., and Mowlavi N. (2004)
The Geneva-Copenhagen survey of the solar neighbourhood. Astron. Astrophys., 418, 989-1019.

Nyquist L., Lindstrom D., Mittlefehldt D., Shih C.-Y., Wiesmann H., Wentworth S., and Martinez R. (2001) Manganese-chromium formation intervals for chondrules from the Bishunpur and Chainpur meteorites. Meteoritics \& Planet. Sci., 36, 911938.

Oey M. S. (2000) A new look at simple inhomogeneous chemical evolution. Astrophys. J. Lett., 542, L25-L28.

Pagel B. E. J. (1989) An analytical model for the evolution of primary elements in the galaxy. Rev. Mexicana Astron. Astrofis., $18,161-172$.

Pagel B. E. J. (1997) Nucleosynthesis and Chemical Evolution of Galaxies. Cambridge Univ., Cambridge. 378 pp.

Penzias A. A. (1981) The isotopic abundances of interstellar oxygen. Astrophys. J., 249, 518-523.

Prantzos N., Aubert O., and Audouze J. (1996) Evolution of the carbon and oxygen isotopes in the galaxy. Astron. Astrophys., 309, 760-774.

Prinzhofer A., Papanastassiou D. A., and Wasserburg G. J. (1992) Samarium-neodymium evolution of meteorites. Geochim. Cosmochim. Acta, 56, 797-815.

Qian Y.-Z., Vogel P., and Wasserburg G. J. (1998) Diverse supernova sources for the r-process. Astrophys. J., 494, 285-296.

Quitté G. and Birck J. (2004) Tungsten isotopes in eucrites revisited and the initial ${ }^{182} \mathrm{Hf} / 180 \mathrm{Hf}$ of the solar system based on iron meteorite data. Earth Planet. Sci. Lett., 219, 201-207.

Raiteri C. M., Villata M., and Navarro J. F. (1996) Simulations of galactic chemical evolution. I. $\mathrm{O}$ and $\mathrm{Fe}$ abundances in a simple collapse model. Astron. Astrophys., 315, 105-115.

Rauscher T., Heger A., Hoffman R. D., and Woosley S. E. (2002) Nucleosynthesis in massive stars with improved nuclear and stellar physics. Astrophys. J., 576, 323-348.

Reddy B. E., Tomkin J., Lambert D. L., and Allende Prieto C. (2003) The chemical compositions of galactic disc F and G dwarfs. Mon. Not. R. Astron. Soc., 340, 304-340.

Reynolds J. H. (1960) Determination of the age of the elements. Phys. Rev. Lett., 4, 8-10.

Rocha-Pinto H. J., Maciel W. J., Scalo J., and Flynn C. (2000) Chemical enrichment and star formation in the Milky Way disk. I. Sample description and chromospheric age-metallicity relation. Astron. Astrophys., 358, 850-868.

Romano D. and Matteucci F. (2003) Nova nucleosynthesis and galactic evolution of the CNO isotopes. Mon. Not. R. Astron. Soc., 342, 185-198.

Rowe M. W. and Kuroda P. K. (1965) Fissiogenic xenon from the Pasamonte meteorite. J. Geophys. Res., 70, 709-714.

Rutherford E. (1929) Origin of actinium and age of the Earth. Nature, 123, 313-314.

Salpeter E. E. (1955) The luminosity function and stellar evolution. Astrophys. J., 121, 161-167.

Scalo J. M. (1986) The stellar initial mass function. Fund. Cosmic Phys., 11, 1-278.

Schatz H., Toenjes R., Pfeiffer B., Beers T. C., Cowan J. J., Hill V., and Kratz K. (2002) Thorium and uranium chronometers applied to CS 31082-001. Astrophys. J., 579, 626-638.

Schmidt M. (1959) The rate of star formation. Astrophys. J., 129, 243-258.

Schmidt M. (1963) The rate of star formation. II. The rate of formation of stars of different mass. Astrophys. J., 137, 758-769.

Schönbächler M., Rehkämper M., Halliday A. N., Lee D.-C., Bourot-Denise M., Zanda B., Hattendorf B., and Gunther D. 
(2002) Niobium-zirconium chronometry and early solar system development. Science, 295, 1705-1708.

Shetrone M., Venn K. A., Tolstoy E., Primas F., Hill V., and Kaufer A. (2003) VLT/UVES abundances in four nearby dwarf spheroidal galaxies. I. Nucleosynthesis and abundance ratios. Astron. J., 125, 684-706.

Sneden C., Cowan J. J., Ivans I. I., Fuller G. M., Burles S., Beers T. C., and Lawler J. E. (2000) Evidence of multiple r-process sites in the early galaxy: New observations of CS 22892-052. Astrophys. J. Lett., 533, L139-L142.

Spergel D. N., Verde L., Peiris H. V., Komatsu E., Nolta M. R., Bennett C. L., Halpern M., Hinshaw G., Jarosik N., Kogut A., Limon M., Meyer S. S., Page L., Tucker G. S., Weiland J. L., Wollack E., and Wright E. L. (2003) First-year Wilkinson Microwave Anisotropy Probe (WMAP) observations: Determination of cosmological parameters. Astrophys. J. Suppl. Ser., $148,175-194$.

Thiemens M. H. and Heidenreich J. E. (1983) The mas independent fractionation of oxygen: A novel isotope effect and its possible cosmochemical implications. Science, 219, 10731075.

Timmes F. X. and Clayton D. D. (1996) Galactic evolution of silicon isotopes: Application to presolar $\mathrm{SiC}$ grains from meteorites. Astrophys. J., 472, 723-741.

Timmes F. X., Woosley S. E., and Weaver T. A. (1995) Galactic chemical evolution: Hydrogen through zinc. Astrophys. J., 98, 617-658.

Tinsley B. M. (1977) Chemical evolution in the solar neighborhood. III - Time scales and nucleochronology. Astrophys. J., 216, 548-559.

Tinsley B. M. (1980) Evolution of the stars and gas in galaxies. Fund. Cosmic Phys., 5, 287-388.

Travaglio C., Galli D., and Burkert A. (2001) Inhomogeneous chemical evolution of the galactic halo: Abundance of r-process elements. Astrophys. J., 547, 217-230.

Twarog B. A. (1980) The chemical evolution of the solar neighborhood. II - The age-metallicity relation and the history of star formation in the galactic disk. Astrophys. J., 242, 242259.

van den Bergh S. (1962) The frequency of stars with different metal abundances. Astron. J., 67, 486-490.

van den Hoek L. and de Jong T. (1997) Inhomogeneous chemical evolution of the galactic disk: Evidence for sequential stellar enrichment? Astron. Astrophys., 318, 231-251.

Vanhala H. A. T. and Boss A. P. (2002) Injection of radioactivities into the forming solar system. Astrophys. J., 575, 1144-1150.

Wakker B. P., Howk J. C., Savage B. D., van Woerden H., Tufte S. L., Schwarz U. J., Benjamin R., Reynolds R. J., Peletier R. F., and Kalberla P. M. W. (1999) Accretion of low-metallicity gas by the Milky Way. Nature, 402, 388-390.
Wasserburg G. J., Busso M., Gallino R., and Raiteri C. M. (1994) Asymptotic giant branch stars as a source of short-lived radioactive nuclei in the solar nebula. Astrophys. J., 424, 412-428.

Wasserburg G. J., Busso M., and Gallino R. (1996) Abundances of actinides and short-lived nonactinides in the interstellar medium: Diverse supernova sources for the r-processes. Astrophys. J. Lett., 466, L109-L113.

Wheeler J. C., Sneden C., and Truran J. W. (1989) Abundance ratios as a function of metallicity. Annu. Rev. Astron. Astrophys., 27, 279-349.

Wielen R., Fuchs B., and Dettbarn C. (1996) On the birth-place of the Sun and the places of formation of other nearby stars. Astron. Astrophys., 314, 438-447.

Wilson T. L. and Rood R. T. (1994) Abundances in the interstellar medium. Annu. Rev. Astron. Astrophys., 32, 191-226.

Woosley S. E. and Weaver T. A. (1995) The evolution and explosion of massive stars, II. Explosive hydrodynamics and nucleosynthesis. Astrophys. J. Suppl. Ser., 101, 181-235.

Wyse R. (2003) Galactic encounters. Science, 301, 1055-1057.

Wyse R. F. G. and Silk J. (1989) Star formation rates and abundance gradients in disk galaxies. Astrophys. J., 339, 700-711.

Yin Q., Jacobsen S. B., McDonough W. F., Horn I., Petaev M. I., and Zipfel J. (2000) Supernova sources and the ${ }^{92} \mathrm{Nb}-{ }^{92} \mathrm{Zr}$ pprocess chronometer. Astrophys. J. Lett., 536, L49-L53.

Yin Q., Jacobsen S. B., Yamashita K., Blichert-Toft J., Télouk P., and Albaréde F. (2002) A short timescale for terrestrial planet formation from Hf-W chronometry of meteorites. Nature, 418, 949-952.

Yokoi K., Takahashi K., and Arnould M. (1983) The Re-187-Os187 chronology and chemical evolution of the galaxy. Astron. Astrophys., 117, 65-82.

Zinner E. (1998) Stellar nucleosynthesis and the isotopic composition of presolar grains from primitive meteorites. Annu. Rev. Earth Planet. Sci., 26, 147-188.

Zinner E., Amari S., Guinness R., Nguyen A., Stadermann F., Walker R. M., and Lewis R. S. (2003) Presolar spinel grains from the Murray and Murchison carbonaceous chondrites. Geochim. Cosmochim. Acta, 67, 5083-5095.

Zinner E., Amari S., Jennings C., Mertz A. F., Nguyen A. N., Nittler L. R., Hoppe P., Gallino R., and Lugaro M. (2005a) $\mathrm{Al}$ and $\mathrm{Ti}$ isotopic ratios of presolar $\mathrm{SiC}$ grains of type $\mathrm{Z}$ (abstract). In Lunar and Planetary Science XXXVI, Abstract \#1691. Lunar and Planetary Institute, Houston (CD-ROM).

Zinner E., Nittler L. R., Hoppe P., Gallino R., Straniero O., and Alexander C. M. O’D. (2005b) Oxygen, magnesium and chromium isotopic ratios of presolar spinel grains. Geochim. Cosmochim. Acta, in press. 